\title{
Generationale Verpflichtungen - normative Muster und ihre Umsetzung
}

\author{
Doris Bühler-Niederberger
}

Online publiziert: 25. Juni 2020

(C) Der/die Autor(en) 2020

Zusammenfassung Das ,generationing“ als Definition von Alterskategorien und Zurechnung von Individuen zu diesen regelt auch Verpflichtungen und Solidaritätserwartungen zwischen den Angehörigen so definierter Gruppen. Sowohl in Gesellschaften des globalen Nordens wie des Südens wird Solidarität wesentlich entlang von Alterslinien geregelt. Schematisierend lassen sich ein Independenz- und ein Interdependenzmodell unterscheiden: Sie erfassen je andere Verteilungen und ihnen zugrundeliegende normative Muster. Im ersten Fall steht eine durch Staat und Experten gestützte Vorstellung ,guter Kindheit“" im Zentrum, im zweiten eine auf Traditionen rekurrierende und gesetzlich gestärkte Vorstellung von „filial piety“. So unterschiedlich die Verteilungen und Verteilungsregeln sind, kommt ihnen doch innerhalb der jeweiligen sozialen und kulturellen Kontexte eine hohe Selbstverständlichkeit und normative Verbindlichkeit zu. Generationale Verpflichtungen sind zwar ordnungsstiftend und schaffen Solidarität, benachteiligen dabei aber auch einzelne Gruppen systematisch im Zusammenspiel von familialen Interessen und staatlichen Regelungen. Der Aufsatz bietet einen systematischen Aufriss der beiden Modelle und bilanziert anhand von Länderbeispielen die Leistungen und Verwerfungen der jeweiligen generationalen Verteilungsregeln und ihrer Umsetzung.

Schlüsselwörter Generation · Alterskategorisierung · Independenzmodell · Interdependenzmodell $\cdot$ Solidarität

D. Bühler-Niederberger $(\bowtie)$

Bergische Universität Wuppertal, Gaußstraße 20, 42119 Wuppertal, Deutschland

E-Mail: buehler@uni-wuppertal.de 


\title{
Generational obligations: normative patterns and material accomplishment
}

\begin{abstract}
Generationing" as a definition of age categories and as the attribution of individuals to them also regulates obligations and expectations of solidarity between the members of groups thus defined. In societies of both the global North and South, solidarity is essentially regulated along age lines. A schematic distinction can be made between an independence and an interdependence model, with each capturing different distributions and underlying normative patterns. In the first case, the focus is on an idea of "good childhood" supported by the state and experts, while in the second, it is on a legally supported idea of "filial piety" that refers back to traditions. As different as distributions and rules of distribution may be, they are taken for granted within the respective social and cultural contexts. Generational obligations do indeed create order and create solidarity, but they also systematically disadvantage individual groups in the interplay of family interests and state regulations. The paper provides a systematic outline of the two models and, based on country examples, takes stock of the achievements and distortions resulting from the respective generational rules of distribution and their implementation.
\end{abstract}

Keywords Generation - Age categorization · Independence model · Interdependence model $\cdot$ Solidarity

\section{Les obligations intergénérationnelles : deux modèles normatifs et leur mise en ouvre}

Résumé Le " generationing », c'est-à-dire la définition de classes d'âge et la catégorisation des individus en fonction de celles-ci, régit les obligations et les attentes en matière de solidarité entre les membres des groupes ainsi définis. Dans les sociétés du Nord comme dans celles du Sud, la solidarité est essentiellement régulée en fonction de l'âge. Schématiquement, on peut distinguer deux modèles : un modèle d'indépendance et un modèle d'interdépendance. Ces modèles renvoient à différentes répartitions sous-tendues par des principes normatifs distincts. Le premier est axé sur la notion de «bonne enfance » soutenue par l'État et les experts ; le second sur la notion de «piété filiale » fondée sur la tradition et renforcée par la législation. Aussi différentes que soient ces répartitions et les règles qui les sous-tendent, elles sont considérées dans leur contexte social et culturel respectif comme allant de soi et ayant valeur d'obligation. Les obligations intergénérationnelles génèrent certes de l'ordre et de la solidarité, mais elles désavantagent aussi certains groupes de manière systématique par le jeu croisé des intérêts familiaux et des réglementations étatiques. Cet article donne un aperçu systématique de ces deux modèles et fait le point sur les forces et les faiblesses des règles de répartition entre les générations qui les constituent à l'aide d'exemples de leur mise en œuvre dans différents pays.

Mots-clés Génération · Catégorisation selon l'âge · Modèle d'indépendance · Modèle d'interdépendance · Solidarité 


\section{Einleitung - Die generationale Ordnung gesellschaftlicher Solidarität}

Das Alter ist eine Dimension, die in der Soziologie vergleichsweise wenig beachtet wird. Zwar werden die Lebenslagen einzelner Altersgruppen - von Kindern, Jugendlichen, Alten - thematisiert, aber ohne dass das Alter die gleiche Aufmerksamkeit als Strukturdimension fände, wie sie dem Geschlecht oder der Schichtzugehörigkeit zuteil wird. Untersucht man jedoch Leistungen der Unterstützung und Umverteilung zwischen Gesellschaftsmitgliedern, so sind diese wesentlich entlang vom Alter und von Altersgruppen strukturiert. Genauer: Die Zugehörigkeit zu einer sozial definierten Alterskategorie ist mit Erwartungen verbunden, die man an die Mitglieder anderer Altersgruppen richten darf und denen man selbst zu entsprechen hat. Bezogen auf das Thema der Solidarität heißt das also, dass Solidarität - verstanden als Leistungen der Unterstützung und Umverteilung - generational geordnet ist; zumindest ist sie dies auch. Bemerkenswert ist, in welch unterschiedlicher Weise sie in verschiedenen Gesellschaften generational geordnet werden kann und wie verpflichtend diese Ordnungen sind.

Zunächst muss man den Begriff der Generation klären, wie er hier verwendet wird. Der Begriff hat innerhalb und außerhalb der Soziologie sehr unterschiedliche Bedeutungen angenommen, die es auseinanderzuhalten gilt. Das hat schon Kertzer (1983) angemahnt. Die älteste Bedeutung ist die der verwandtschaftlichen Abstammung, der Deszendenz, im Sinne von Eltern-Kind-Beziehungen, aber auch von umfassenderen verwandtschaftlichen Beziehungen. Die Sozialwissenschaften verwenden den Begriff jedoch auch zur Bezeichnung von Kohorten (d.h. für Personen, die im gleichen Zeitintervall geboren bzw. mit wichtigen Ereignissen konfrontiert wurden), von Lebensabschnitten und von historischen Perioden. Und nicht selten verwendet ihn ein und derselbe Soziologe mit verschiedenen Konnotationen: Während Durkheim (1972) ihn meist als Kohorte versteht, bezieht sich Eisenstadt (1956) sowohl auf Lebensabschnitte als auch auf verwandtschaftliche Abstammung. Und für Mannheim (1964) hat er eine genealogische Bedeutung, hauptsächlich aber die Bedeutung der Kohorte, wobei er ein besonderes Gewicht auf deren gemeinsame Erfahrungen legt (Kertzer 1983, S. 127). Es ist also nicht ganz unproblematisch, wenn der Generationenbegriff in diesem Beitrag noch in einer weiteren, davon abweichenden Bedeutung verwendet wird, aber diese Begriffsverwendung ist inzwischen bereits so weit in die Diskurse insbesondere der Kindheitsforschung eingedrungen, dass eine andere Bezeichnung für das hier Gemeinte noch verwirrender wäre.

Unter Generation soll nachfolgend das sozial definierte Alter im Unterschied zum chronologisch-biologischen Alter verstanden werden - in Analogie zum Begriffspaar Gender und Sex. Ausgangspunkt für dieses Verständnis ist das Konzept des „generationing“, mit dem Leena Alanen (2001) den gesellschaftlichen Prozess der Definition von Alterskategorien adressiert. Im Zuge dieses Prozesses werden Kategorien des Alters gebildet, institutionalisiert und gewertet; das ist die Makroebene des Geschehens. Die Gesellschaftsmitglieder rechnen sich in ihren Interaktionen selbst und wechselseitig solchen Kategorien zu; das ist die Mikroebene des Geschehens. Alanen und Mayall (2001) thematisieren diesen Prozess der gesellschaftlichen Interpretation und Übersetzung des chronologischen Alters in soziale Alterskategorien, weil darin über die Verteilung von Ressourcen zwischen den so definierten 
Altersgruppen entschieden wird, unterschiedliche materielle und immaterielle Teilhabeberechtigungen festlegt werden, ,voice“ zugestanden oder verweigert wird und der Zugang zu physischen und gesellschaftlichen Räumen reguliert wird. Dabei gilt ihr Interesse den asymmetrischen Verteilungen von Rechten, Pflichten und Ressourcen zwischen den als „Kinder“ und als „Erwachsene“ Kategorisierten: „righting children's wrongs“ (Mayall 2000, S. 246), das Durchsetzen von Rechten für Kinder, ist ihr Anliegen. In dieser Bedeutung hat der Begriff des ,generationing“ in der soziologischen Thematisierung von Kindheit schnell und in verschiedenen Ländern Karriere gemacht (Bühler-Niederberger 2010). In dem nachfolgenden Beitrag steht dagegen zunächst nicht die relative Benachteiligung einer der so geschaffenen Altersgruppen im Vordergrund, sondern die allgemeine Tatsache, dass Solidarität zwischen den Gesellschaftsmitgliedern in einem hohen Grade generational, d.h. entlang der sozial definierten Alterskategorien geordnet wird.

Der Aspekt der Ungleichheit bleibt gleichwohl relevant. Generational geordnete Verteilungen und also auch die generational begründete Solidarität folgen in der Regel keinen Vorstellungen von Gleichverteilung, sie sind nicht per se gerecht. Als gerechtfertigt, ja weitgehend selbstverständlich gelten sie durch die quasi-natürliche Grundlage, die das Alter abgibt, wenn es darum geht, die Verpflichtungen und Berechtigungen, die konkreten Individuen und Personengruppen zustehen, zu definieren. Sie verdanken ihre Geltungskraft auch der Verschränkung von Alterskategorisierung und Geschlechtskategorisierung, wie noch zu zeigen sein wird. Und sie werden zusätzlich legitimiert durch starke normative Muster, die Abweichungen als ernstzunehmenden moralischen, oft aber auch rechtlich $\mathrm{zu}$ ahndenden Verstoß brandmarken. Auch wenn die in diesem Beitrag angesprochenen Leistungen der Unterstützung und Umverteilung vor allem im Rahmen von Familien und Verwandtschaft erbracht werden, greifen sie darüber hinaus, und die staatlichen Institutionen sind an ihrer Regelung und Legitimation beteiligt.

Die grundsätzliche Selbstverständlichkeit generational geordneter Verteilungen ist erstaunlich, wenn man die empirische Variation geltender Verteilungsregeln und praktizierter Verteilungen betrachtet. In diesem Beitrag sollen zwei grundlegend verschiedene Modelle vorgestellt werden, in die sich die meisten generationalen Verteilungssysteme einordnen lassen. Man kann den Unterschied der Modelle vorerst grob so skizzieren: Im ersten Modell steht die Sorge für die heranwachsenden (und zunehmend auch für die erwachsenen) Kinder im Mittelpunkt der Familie. Die Verpflichtung, eine ,gute Kindheit“ im Sinne eines behüteten und geförderten Aufwachsens im Rahmen der Kernfamilie zu gestalten, absorbiert einen wesentlichen Anteil der materiellen und immateriellen Ressourcen der Familie. Diese grundsätzliche Verpflichtung gilt in den bürgerlichen Familien westlicher Gesellschaften bereits seit dem Beginn der Neuzeit, und ab dem beginnenden 20. Jahrhundert gilt sie für alle Bevölkerungsgruppen, auch aufgrund massiver Interventionen von Staat und Experten. Sie gilt als Leitbild ungebrochen bis heute, und die insbesondere von den Eltern verlangte Leistung wächst beständig (Ariès 1978; Schlumbohm 1983; Zelizer 1985; Bühler-Niederberger 2020a). Im zweiten Modell sind es der Respekt und die Aufmerksamkeit der Kinder für die alten Eltern und überhaupt für alte Verwandte, auch bezeichnet als ,filial piety“, die im Zentrum des Familienlebens zu stehen haben. Das gilt in den meisten Ländern Asiens und Afrikas und in weiten Teilen der 
Bevölkerung Lateinamerikas. Diese Anforderungen haben sich inzwischen in den modernen asiatischen Ländern und unter den Bedingungen der Migration zum Teil verändert; vor allem wurden die Beziehungen zwischen den jüngeren und älteren Generationen gleichberechtigter und demokratischer, ohne dass jedoch die Verpflichtung zu Respekt und zur Unterstützung grundsätzlich ihre Bedeutung verloren hätte (Sharma und Kemp 2012; Yeh et al. 2013; Qi 2015). Man kann die Verteilungsmuster zwischen den Generationen und Altersgruppen - auch jene, die über den Familienkreis hinausgehen - jeweils als rund um diese beiden normativen Kerne angeordnete verstehen; auch die Regelungen der Staaten orientieren sich daran. Keineswegs sollte man die Unterscheidung der beiden Modelle als eine Unterscheidung von Phasen einer historischen Entwicklung verstehen: Beide hier idealtypisch unterschiedenen Konstellationen von Verteilungsregeln und -praktiken haben ihre eigene Geschichte und - soweit sich das zurzeit absehen lässt - auch ihre eigene Zukunft.

Im zweiten Abschnitt des Beitrages werden die beiden Modelle auf der Basis verschiedener, darunter auch eigener empirischer Forschungsergebnisse zu normativen Verteilungsregeln und realen Verteilungsmustern hergeleitet. Mit der Heranziehung von Forschungsergebnissen aus unterschiedlichen Ländern wird das Ziel verfolgt zu zeigen, wie global verbreitet generationale Verteilungsregeln sind. Sie weisen allerdings im Detail Abweichungen von den idealtypischen Modellen auf, die ebenfalls thematisiert werden sollen, erlauben aber dennoch die weitgehende Zuordnung der Verteilungspraktiken zu einem der beiden Modelle. Die Skizzierung der beiden Modelle ist damit zum einen als Forschungsheuristik zu verstehen, die in ihrer Systematik typische Muster erfasst, aber auch Abweichungen davon sichtbar machen kann, deren Ursachen weiter nachgegangen werden kann. Die beiden Modelle bieten aber auch einen Zugang zu den Alltagsheuristiken der Beteiligten selbst, die sich so die noch zu erhärtende Behauptung - an dem einen oder anderen Modell orientieren, wenn sie die generationalen Verhältnisse und Austauschbeziehungen innerhalb ihrer Familien oder Gesellschaften beurteilen und deren Regeln ggf. teilweise modifizieren.

An welchen Mustern und welchen darin implizierten Verteilungsregeln sich die Akteure auch orientieren: beide Modelle sind ordnungsstiftend und strukturieren Solidarität. Gemeinsam ist ihnen aber auch, dass im Rahmen dieser Ordnungsmodelle einzelne Gruppen systematisch benachteiligt werden. Das soll im dritten Abschnitt herausgearbeitet werden. Über die Benachteiligung resp. Begünstigung bestimmter Familienmitglieder bzw. Alters- und Geschlechtergruppen wird nicht nur auf der Ebene der Familien entschieden, sondern darüber entscheiden auch nationalstaatliche Regelungen. Die typischen Benachteiligungen im Rahmen des ersten Modells sollen vor allem am Beispiel Deutschlands und die im Rahmen des zweiten Modells am Beispiel Kirgisistans ${ }^{1}$ diskutiert werden. Das Fazit zieht eine aktuelle Bilanz über das jeweilige Ausmaß der unterstützenden innerfamilialen und sozialstaatlichen Leistungen und über die Variabilität, Verwerfungen und Gestaltbarkeit von

\footnotetext{
${ }^{1}$ In der zentralasiatischen Republik Kirgisistan, ehemals Teil der Sowjetunion, forscht und lehrt die Verfasserin seit über zehn Jahren mit einiger Regelmäßigkeit. Daraus resultierten wichtige Einsichten in jene Regeln, die hier dem Interdependenzmodell zugerechnet werden.
} 
Verteilungsregeln und -praktiken, die entlang von sozialen Alterskategorien definiert werden.

\section{Modelle generationaler Solidarität}

Im diesem Abschnitt wird versucht, das empirische Wissen über Verteilungen und Unterstützungsleistungen zwischen den Angehörigen verschiedener Alterskategorien in einer Systematik anzuordnen, in der zwei prinzipielle Lösungen für die Regelung dieser Transfers unterschieden werden. Dabei werden die Leistungen über den gesamten Lebenslauf hinweg berücksichtigt; es soll also nicht nur ein Ausschnitt der Generationenbeziehungen betrachtet werden, wie etwa der Aufwand, den Eltern für das Aufziehen ihrer Kinder leisten, oder die Verbundenheit der erwachsenen Kinder mit ihren Herkunftsfamilien oder schließlich die Leistungen der erwachsenen Kinder für ihre alten Eltern. Das folgt dem eingangs angekündigten Anliegen, die grundlegende Relevanz des Alters als gesellschaftlicher Strukturdimension evident zu machen, die über die Thematisierung einzelner Lebensphasen und ihrer jeweiligen Probleme hinausgeht.

Dreierlei muss für diese Systematik geleistet werden: (1) Es müssen Ergebnisse aus unterschiedlichen Forschungsbereichen aufeinander bezogen werden, da die einzelnen Lebensalter prinzipiell unter je anderen Gesichtspunkten und mit anderen Zugängen erforscht wurden. Soweit es um Eltern-Kind-Beziehungen bei Kindern im Kindesalter geht, handelt es sich um Beiträge aus dem Gebiet der Sozialisationsforschung (z. B. Lareau 2003; Vincent und Ball 2007); seit einiger Zeit kommen Ergebnisse aus den „Childhood Studies“ dazu, vor allem auch soweit es um Länder des globalen Südens geht (z. B. Woldehanna et al. 2008; Hunner-Kreisel und Bohne 2016; Punch und Vanderbeck 2018). Was die wechselseitigen Leistungen zwischen erwachsenen Kindern und ihren Eltern betrifft, stammen die herangezogenen Forschungsergebnisse aus recht unterschiedlichen Quellen, je nachdem auf welche Länder sich die Untersuchungen beziehen. Für die westlichen Länder stammen viele Einsichten aus der Alters- und Sozialstaatsforschung (z. B. Szydlik 2016), aber auch aus einer (sich damit überschneidenden) Forschung rund um den Generationenkonflikt, die mit großen Surveystudien arbeitet (z. B. Arber und AttiasDonfut 2000). Für die Länder des globalen Südens, aber auch für industrialisierte Länder Asiens, stammen sie aus Studien zu Alter, Globalisierung, Migration, die oft ethnographisch arbeiten (z. B. Cole und Durham 2007; Lamb 2009), sowie aus qualitativen und quantitativen Studien zur „filial piety“ (z. B. Koyano 1996; Gruijters 2016). Dabei kann selbstverständlich nicht beansprucht werden, einen vollständigen Überblick über all diese Forschungsgebiete und ihre Ergebnisse zu geben. Die Systematik ist als Versuch zu verstehen, die verbreitet geltenden Muster von generational geordneten Verteilungen und Umverteilungen aufzuzeigen und gedanklich zu ordnen. Dieser Anspruch wurde höher gewichtet als eine denkbare weitere Ausdifferenzierung der Systematik, um Abweichungen und Varianten umfassend Rechnung tragen zu können.

(2) Gleichwohl sollen auch Abweichungen von den zwei hier idealtypisch herausgearbeiteten Modellen aufgezeigt werden, und nach Möglichkeit soll auch nach 
deren Zustandekommen gefragt werden. Jedenfalls soll dies exemplarisch geschehen. Eine Funktion dieser Heuristik soll es ja auch sein, auf Bemühungen bestimmter Akteure zu einer Modifikation der einmal eingespielten Muster aufmerksam zu machen. Die Sichtung der unter (1) genannten Forschungserträge macht dies möglich.

(3) Werden die angesprochenen Solidaritätsleistungen auch zum großen Teil in der Familie erbracht, so beschränken sie sich jedoch nicht darauf; bestimmte Leistungen können auch mit einer mehr oder weniger starken Unterstützung durch Staat und Öffentlichkeit einhergehen und sich auf Nichtfamilienangehörige erstrecken. Selbst wenn sie nur den eigenen Familienangehörigen zugedacht sind, werden sie längst nicht nur durch die jeweilige Familie in ihrer Art und Angemessenheit festgelegt. Die Austauschleistungen rekurrieren auf kollektive Wissens- und Wertebestände und sind entsprechend überpersönlich und zum Teil auch gesetzlich festgelegt. ${ }^{2}$ Auch dieser Aspekt soll aus den folgenden Ausführungen ersichtlich werden, auch wenn dafür ebenfalls keine umfassende Bestandsaufnahme geleistet werden kann.

Die beiden Modelle, die hier kontrastiv konstruiert werden, um die Fülle von Untersuchungsergebnissen zu ordnen, sollen als Independenz- und Interdependenzmodell bezeichnet werden. Die Terminologie von Independenz versus Interdependenz ist in der „cultural psychology“ gebräuchlich zur Bezeichnung von stärker individualisierten versus stärker kollektivistischen Gesellschaften (Markus und Kitayama 1991; Triandis 2001). Die Bezeichnung ist allerdings normativ aufgeladen und teilweise irreführend, denn die Verteilungsregeln und -praktiken, die den beiden Modellen zugeordnet werden können, schaffen gleichermaßen Solidarität wie sie auch Benachteiligungen erzeugen, die bis hin zum Ausschluss bestimmter Personen von Solidaritätsleistungen gehen können. Keines der Modelle ist also a priori stärker durch soziale Verpflichtungen zu Unterstützung und Solidarität gekennzeichnet. Unterschiedlich ist dagegen das Ausmaß, in dem die Nationalstaaten bei ihrer Beteiligung an diesen Verteilungsprozessen die Ansprüche Einzelner berücksichtigen oder sie auf ihre Familien verweisen. Das rechtfertigt dann doch die gewählte Begrifflichkeit. Tabelle 1 präsentiert die beiden Modelle im Hinblick auf den materiellen und immateriellen Austausch zwischen den Generationen in bestimmten Lebensphasen. Fokussiert wird die Deszendenzrelation, da sich die generational geordneten Leistungen vorrangig darauf beziehen und hierzu die meisten Untersuchungen vorliegen.

\subsection{Austauschbeziehungen zwischen Erwachsenen und Kindern im Kindesalter}

Die Austauschbeziehungen können entlang der einzelnen Felder in der Tabelle beschrieben und dabei die Unterschiede zwischen den beiden Modellen verdeutlicht werden. Beginnt man mit den Leistungen, die von den Kindern erwartet werden, kann man die Art und den Umfang der Arbeitsbelastung betrachten. Studien zu Deutschland zeigen ein Kindheitsmuster, das zweifellos dem Independenzmodell zuzurechnen ist und fast ausschließlich auf Schule und Freizeit konzentriert ist (Lang

\footnotetext{
2 Einen eindrücklichen Beleg liefert die Forschung zum „,value of children“ (z. B. Nauck 2007; Kağıtçıbaş1 2007): Die Wertzuschreibungen emotionaler, ökonomischer, familistischer Art, die die gesellschaftlichen Kontexte an Kindern vornehmen, erklären die Fertilitätsentscheidungen weit besser als die Zuschreibungen der einzelnen Familien.
} 
Tab. 1 Zwei Grundmuster generational geordneter Solidarität (Quelle: Eigene Darstellung)

\begin{tabular}{llll}
\hline Transfer & \multicolumn{1}{l}{ Von Kindern an Eltern } & Von Eltern an Kinder & Normatives Muster \\
\hline \multicolumn{2}{l}{ Independenzmodell - Kinder im Kindesalter } & \\
Materieller & (1) Kind arbeitet für & (2) Hohe Investition an Zeit & (3) „Verantwortete Eltern- \\
Transfer; & seine eigene Qualifi- & und Geld in die persönliche & schaft“ resp. normatives \\
Care/ & kation; nur minimale & Entwicklung des Kindes; Ver- & Muster der ,guten Kindheit“; \\
Arbeit & zusätzliche Aufgaben & pflichtung, die (besonderen) & Entwicklung des Kindes im \\
& & Fähigkeiten und Neigungen & Zentrum von Familien und \\
& & des Kindes zu fördern & öffentlichen Institutionen
\end{tabular}

Independenzmodell - erwachsene Kinder

Materieller (4) Immaterielle und

Transfer; gelegentlich auch ma-

Care/ terielle Leistungen an

Arbeit alternde Eltern bei deren

Bedarf und entspre-

chend eigenen Ressourcen
(5) Häufig beträchtliche Unterstützung an erwachsene Kinder mit Geld, Unterbringung, bei Hausbau, Betreuung der Enkel usw., zur Vermeidung von sozialem Abstieg oder Erleichterung von Aufstieg

Interdependenzmodell - Kinder im Kindesalter

Materieller (7) Oft hohe Arbeits-

Transfer; last auch jenseits der

Care/ eigenen Qualifikati-

Arbeit on; Kinder oft stark integriert in die Familienwirtschaft; hohe Leistungserwartungen
(8) Eltern ,schenken das Leben“, ernähren das Kind, ziehen es groß und leisten die moralische Erziehung
(6) Kein normatives Muster. Vor allem spontane Transfers, zumeist in Kombination mit wohlfahrtsstaatlichen Umlageverfahren
(9) „Filial piety“; Kinder sind von klein auf und lebenslang zu Dankbarkeit, Respekt und Unterstützung verpflichtet

\section{Interdependenzmodell - erwachsene Kinder}

Materieller (10) Oft hohe Arbeits-

Transfer; last, v. a. für SchwiegerCare/ töchter; oft Verpflich-

Arbeit tung zur virilokalen Residenz; Geldrücksendungen; Leistungen als Pflicht und Respekt
(11) Erbe, Haus (oft an den jüngsten Sohn); verpflichtend angebotene Koresidenz; u. U. Sorge für Enkel, z. B. wenn die Kinder migrieren; wenig Unterstützung an verheiratete Töchter
(12) „Filial piety“; Unterstützungsverpflichtung wird u. U. auch durch den Staat bekräftigt resp. gesetzlich eingefordert; diverse, oft ungenügende öffentliche Altersversorgungssysteme

1985; Zinnecker und Silbereisen 1996; LBS 2009; World Vision 2018). Das gilt im Wesentlichen auch für andere westliche Länder: Eine Studie von Bruckauf und Rees (2017) zeigt, dass etwa die Hälfte der Schulkinder in zehn EU- und OECD-Ländern nur selten oder nie das tun, was ,zu Hause helfen“ genannt wird (Feld 1).

Demgegenüber zeigen Studien und Statistiken über Kinder in Ländern des globalen Südens das große Arbeitspensum, das sie vor allem in ländlichen Gegenden bewältigen (z.B. Punch 2001; Jacquemin 2004; Durham 2007; Woldehanna et al. 2008). Ihre Arbeit ist in die Gesamtökonomie des Haushalts und der Familie fest integriert, das heißt, die Kinder wissen, was es zu tun gilt, warum es dies zu tun gilt und wie es in die Existenzsicherung eingebunden ist, und sie sind initiativ mit ihren Arbeitsbeiträgen (Coppens et al. 2018) (Feld 7).

Ein weiterer Aspekt mag diese unterschiedlichen Arbeitsleistungen, die die Kinder erbringen, verdeutlichen: die Frage nämlich, bei wem die Kinder leben. In Ländern des globalen Südens, aber auch in Übergangsgesellschaften, wie z. B. China, sind dies bei einem beachtlichen Teil nicht die leiblichen Eltern oder Elternteile 
(Lu 2012; Crivello und Espinoza-Revollo 2018). Besonders hoch ist der Anteil der Kinder, die nicht bei ihren Eltern aufwachsen, in den Ländern Afrikas; so wohnen nach Angaben von Bray und Dawes (2016) ein Viertel bis ein Drittel der Kinder ab 10 Jahren mit keinem Elternteil zusammen. Wichtige Gründe dafür sind die Arbeitsmigration der Eltern innerhalb des Landes oder ins Ausland und ein früher Tod der Eltern. Das Leben in einem anderen Haushalt kann aber auch dadurch begründet sein, dass in diesem die Arbeitskraft der Kinder und Jugendlichen gebraucht wird.

Diese Arbeitsverpflichtung für die Kinder im Haushalt, im Betrieb oder außerhalb der Familie fällt vermutlich geringer aus - Vergleichsdaten gibt es leider kaum -, wenn die Länder reicher und urbaner sind, gleichwohl aber aufgrund ihrer generationalen Verteilungsregeln insgesamt dennoch dem Interdependenzmodell zugerechnet werden können. Ein solcher Fall ist Südkorea, das eines der untersuchten zehn Länder in der bereits zitierten Studie von Bruckauf und Rees (2017) war. Zwar gibt es hier einen besonders kleinen Prozentsatz von Kindern, die angeben, sich nie an Hausarbeit zu beteiligen; zugleich ist aber auch die Zahl der Kinder, die angeben, das täglich zu tun, auffallend niedrig. Recht hoch ist dagegen die Arbeitsleistung der Kinder in China. Hier arbeiten laut einer aktuellen Studie die Mädchen immer noch fast fünf Stunden pro Woche im Haushalt, die Jungen mehr als drei Stunden; die Variable Stadt/Land erwies sich in der multivariaten Analyse dabei als wenig erklärungskräftig (Hu 2018). Diese Arbeitsleistung kontrastiert sehr deutlich mit den anderthalb Stunden bzw. der einen Stunde, die gleichaltrige deutsche Mädchen bzw. Jungen laut einer Zeitbudgetstudie (BMFSFJ 2006) wöchentlich für die Mithilfe im Haushalt aufwenden. Hoch sind auch die Erwartungen an den Bildungserfolg der Kinder, wenn die Verteilungsregeln des zweiten Modells zur Anwendung kommen. Das zeigt Woronow (2007) in einer Studie über Erziehungsratschläge und -praktiken in China.

Die Unterschiede zwischen den Erwartungen an das Kind respektive den Beiträgen des Kindes im Rahmen des Independenz- und des Interdependenzmodells (Felder 1 und 7) können durch Ergebnisse der eigenen Forschung in Kirgisistan verdeutlicht werden. Hinsichtlich seiner wirtschaftlichen Daten kann man Kirgisistan als ein Land des globalen Südens betrachten. Da das Land zur Sowjetunion gehörte, weist es aber bereits in der Eltern-, ja der Großelterngeneration eine vollständige Alphabetisierung auf, ist also in dieser Hinsicht sicher kein „Entwicklungsland“. Was die Arbeitsleistungen der Kinder betrifft, waren sie bereits bei den Kindergartenkindern erstaunlich hoch. Bei insgesamt über 100 Vorschulkindern in städtischen und ländlichen Regionen wurden mit kindgerechten Erhebungsmethoden Daten zur Familien- und Arbeitssituation gesammelt, die insgesamt den Eindruck einer starken Einbindung der Kinder in die ihnen zugedachten Verpflichtungen ergaben. So ließen wir 61 Kinder beim Einrichten eines Puppenhauses erzählen, wie ihr Tagesablauf aussieht. Nur 9 dieser Drei- bis Sechsjährigen nannten keine häuslichen Aufgaben, die sie im Laufe des Tages übernehmen, die übrigen zählten folgende Leistungen auf: Wasser holen, sauber machen im und um das Haus, Feuerholz suchen, kleinere Geschwister hüten, in der Küche helfen, den Tee auftischen (Bühler-Niederberger und Schwittek 2014). Soweit es die Erwartungen der Eltern hinsichtlich der Schulleistung ihrer Kinder betrifft bzw. die eigene Leistungsbereitschaft der Kinder, können Ergebnisse aus einer Befragung von jeweils gut 400 Zweit- und Drittklässlern in 
Kirgisistan und in Deutschland herangezogen werden. „In der Schule möchte ich die Beste/der Beste sein“, war eine Aussage, von der $88 \%$ der kirgisischen Kinder sagten ,stimmt voll und ganz“. Dagegen stimmten dem nur 39\% der deutschen Kinder vollumfänglich zu. Vergleichbare Unterschiede fanden wir bei den Berufswünschen: Die kirgisischen Kinder wünschten sich fast ausnahmslos akademische Berufe, und sie begründeten dies oft mit der Unterstützungsleistung für ihre Familie, die das später ermögliche; die deutschen Kinder wählten Berufe auf verschiedenen Anspruchsniveaus und stark orientiert an ihren Lieblingsbeschäftigungen und den Fähigkeiten, die sie sich selbst zuschrieben (Bühler-Niederberger 2020b).

Den unterschiedlichen Leistungen der Kinder entsprechen unterschiedliche Verpflichtungen der Eltern gegenüber ihren Kindern. Für Eltern westlicher Länder gilt die Norm, dass die Kinder bei der Entwicklung ihrer Begabungen und Neigungen unterstützt werden sollen. François de Singly spricht sogar von einem kategorischen Imperativ: „Das Kind muss die Möglichkeiten bekommen, sich selbst zu werden“ (2005, S. 108; Übers. D. B.-N.). Das erfordert hohe Investitionen von den Eltern: an Zeit, Zuwendung, materieller Ausstattung. Der wachsende Markt an Konsumgütern für Kinder ist nicht zu übersehen, man kann aber auch auf weniger beachtete Erscheinungen hinweisen: In den vergangenen Jahren wurden in verschiedenen Studien in westeuropäischen Ländern und den USA die Kurse und Vereinsmitgliedschaften erfasst, die die Eltern für ihre Kinder organisieren und bezahlen. Sie sind nicht gleichzusetzen mit der „shadow education“, die klar auf den Bildungserfolg ausgerichtet ist (Entrich 2015). Dadurch sollen vor allem künstlerische und sportliche Begabungen entdeckt und gefördert werden. Die Kinder kommen zum Teil schon im Vorschulalter in den Genuss solcher Angebote, oft werden mehrere Angebote gleichzeitig in Anspruch genommen. Die Kurse werden nicht selten nach kurzer Zeit abgewählt und durch neue ersetzt, die den Interessen und Fähigkeiten der Kinder vielleicht besser entsprechen mögen (Lareau 2003; Vincent und Ball 2007; Bischoff-Pabst und Knoll 2015; World Vision 2018). Eine wichtige Funktion in dieser Kindheit als einer Phase der Selbstfindung und Selbstkultivierung hat auch das Haustier. Es wird von Experten angepriesen: Es sei nicht nur ein Vergnügen für das Kind, sondern fördere seine Fürsorglichkeit und emotionale Qualitäten. Mehr als zwei Drittel der Kinder in Deutschland besitzen ein Haustier, und zwar im Durchschnitt gleich zwei verschiedene Tierarten. Es bedeutet ihnen sehr viel und stellt eine weitere Arbeit für die Mutter dar (Zinnecker et al. 2003; Muldoon et al. 2014). Diese intensiv fördernde Zuwendung zum Kind, für die die Experten das neue Wort ,parenting“ (Ostner et al. 2017) geschaffen haben, wird durch ein normatives Muster geleitet, das man als „,verantwortete Elternschaft“ bezeichnen kann oder als „gute Kindheit“. Gemeint ist eine starke Verpflichtung der Eltern, die bestmöglichen Umstände für die optimale Entwicklung ihrer Kinder zu schaffen, ja das ganze Familienleben auf dieses Ziel hin zu strukturieren (Nave-Herz 2015; Schneider et al. 2015; Bühler-Niederberger 2020a) (Felder 2 und 3).

Im Interdependenzmodell sind die Verpflichtungen und Verdienste der Eltern anders definiert. Als Spender des Lebens verdienen Eltern die lebenslange Dankbarkeit ihrer Kinder, wie Magazine und Ramirez Sanchez dies für die ländliche Bevölkerung in Mexiko analysiert haben: Die Kinder werden nicht in einen Naturzustand geboren, sondern in einen „state of indebtedness“ (2007, S. 62); diese Schuld müs- 
sen sie zurückerstatten. Es wird von ihnen erwartet, dass sie arbeiten, um die Schuld abzutragen und die Aufwendungen zu begleichen, die gemacht werden mussten, um sie zu ernähren und großzuziehen; das zeigen auch andere Studien in verschiedenen Ländern und Kontinenten des globalen Südens (Durham 2007; Cole und Durham 2007; Woldehanna et al. 2008.). Das wird nicht nur auf der Ebene der Familien so definiert; öffentliche Institutionen wie die Schule oder die religiösen Einrichtungen verkünden dieselben Botschaften. Im gleichen Tenor argumentiert die „African Charter on the Rights and Welfare of the Child", ${ }_{3}$ die die afrikanischen Staaten als Ergänzung zur UN-Kinderrechtskonvention verfasst haben. Im Artikel 31 hält sie auch die Verantwortlichkeiten der Kinder fest: den Zusammenhalt der Familie zu befördern, ihren Eltern beizustehen sowie Eltern, Ältere und Vorgesetzte zu respektieren. Wie Magazine und Ramirez Sanchez (2007) ausführen, kann auf der Grundlage dieses Verständnisses von Schuld und Dankbarkeit ein lebenslanger Zyklus des Austausches von Unterstützungsleistungen zwischen Eltern und Kindern in Gang gesetzt werden. Bray und Dawes zeigen in einem Überblick über Studien im Süden und Osten Afrikas, dass dort eine Vorstellung des ,parenting“, und also auch zum Beispiel von verschiedenen Erziehungsstilen, nicht existiert (2016, S. 5); sie wird von außen an diese Länder herangetragen. Auch aus der Sicht der Heranwachsenden steht vielmehr die Reziprozität im Vordergrund im Sinne von gegenseitigen Unterstützungsleistungen zwischen Eltern und Kindern (Felder 8 und 9).

Wie unterschiedlich die Beiträge der Eltern und die ihnen zugrundeliegenden normativen Muster sind, kann auch anhand der eigenen vergleichenden Forschung illustriert werden. In einer Studie wurden 40 kirgisische Studierende gebeten, den Satzanfang „Das Wichtigste, was mir meine Eltern für das Leben mitgaben ...“ zu ergänzen. Sie schrieben dabei Sätze wie diese: „Sie gaben mir das Leben. Sie lehrten mich, den richtigen Weg zu wählen, sie lehrten mich Alles: gute Taten von schlechten zu unterscheiden. Sie gaben mir alle die wunderbaren Teile meines Körpers. Ich habe Arme, Beine, Augen, eine Nase.“ Oder: „Das Wichtigste, was meine Eltern mir gaben, ist mein Leben. Sie haben mich gut erzogen, mir immer gesagt, ehrlich zu sein." Nur vier der 40 Studierenden sprachen von so etwas wie Liebe, Wärme oder emotionalem Austausch. Fast ausnahmslos drückten sie also ihren Dank aus für das ihnen geschenkte Leben, das Aufziehen und die moralische Unterweisung durch die Eltern. Ganz anders antworteten 40 Studierende aus den Niederlanden. Sie lobten die Liebe und Wärme, die sie erhalten hatten, oder sie dankten für die Unterstützung bei der Umsetzung ihrer eigenen Wünsche und Pläne, für die Ermutigung und Rückendeckung bei der Wahl des eigenen Weges. Alle niederländischen Studierenden hoben die Zuwendung oder die individuelle Unterstützung hervor - mit einer Ausnahme: Einer der Studierenden fand kein gutes Wort für seine Eltern, seine Kindheit sei „unglücklich“ gewesen (Bühler-Niederberger 2020c). Dieselben Unterschiede lassen sich beim Vergleich der kirgisischen Studierenden mit deutschen und italienischen Studierenden konstatieren (Bühler-Niederberger 2020b).

\footnotetext{
3 https://www.achpr.org/public/Document/file/English/achpr_instr_charterchild_eng.pdf. Zugegriffen: Febr. 2020.
} 


\subsection{Austauschbeziehungen zwischen Eltern und erwachsenen Kindern}

Transferleistungen zwischen bereits erwachsenen Kindern und ihren Eltern sind in Ländern, in denen das Independenzmodell vorherrschend ist, nicht nur keine Ausnahme, sondern sogar häufig. Ins Auge stechen die enormen Leistungen älterer Erwachsener an ihre bereits erwachsenen Kinder oder Enkel. Das ist kein neues Phänomen, sondern kann einige Jahrzehnte zurückverfolgt werden (Roussel 1976; Segalen 1990; Arber und Attias-Donfut 2000; Attias-Donfut und Wolff 2000b; Attias-Donfut und Segalen 2002; Kohli et al. 2000; Szydlik 2016). Dieser Fluss von Unterstützungsleistungen von „oben“ "nach ,unten“ in der Altersabfolge soll sozialen Abstieg vermeiden und Aufstieg ermöglichen. So wird z. B. die elterliche Hilfeleistung gesteigert bei einer Scheidung oder Trennung der erwachsenen Kinder; sie ist aber auch hoch, wenn die erwachsenen Kinder sich in einer langen Ausbildung befinden (Attias-Donfut und Wolff 2000b). Soweit es um finanzielle Transferleistungen geht, übertrifft der Geldfluss von den Eltern an die erwachsenen Kinder klar den Geldfluss in die umgekehrte Richtung. Diese Aussage lässt sich anhand einer in Frankreich durchgeführten Dreigenerationen-Studie belegen und differenzieren. Attias-Donfut und Wolff (ebd.) untersuchten die Leistungen, die die sogenannte PivotKohorte (geboren zwischen 1939 und 1943) über fünf Jahre hinweg in zwei Richtungen erbrachte: an ihre alten Eltern und an ihre bereits erwachsenen Kinder. In beide Richtungen waren die Hilfeleistungen erheblich, auch aufgrund der geringfügigen anderweitigen Altersversorgung, die den Eltern der Pivot-Kohorte zukam.

Eine sozialstaatliche Alterssicherung - meist als Umlagesystem organisiert - reduziert diesen Transfer nicht. Ganz im Gegenteil ermöglicht und unterstützt der staatlich organisierte Transfer diesen Austausch zwischen den Generationen und ist deshalb auch wichtig für das Wohlergehen der jüngeren Altersgruppen: der erwachsenen Kinder und der Enkelkinder. Attias-Donfut und Wolff (2000a) sprechen von einer Komplementarität von öffentlichen und privaten Transfers. Ebenfalls in einer Dreigenerationen-Studie zeigten Attias-Donfut und Lapierre (1997), wie die Einführung öffentlicher Alterspensionen in Guadeloupe dazu führte, dass sich der Fluss der Solidarität in der Richtung änderte: Privat flossen nun mehr Geld und Sorgeleistungen von den Älteren zu den Kindern und Enkeln als umgekehrt, und dies in einem größeren Umfang, als sie ihrerseits damals von der ältesten Generation bekommen hatten. Andere Studien zeigen, wie die öffentlichen Transferleistungen es den älteren Leuten ermöglichen, weiterhin erhebliche Sparanstrengungen zu unternehmen, um das Erbe zu vergrößern, das sie den erwachsenen Kindern hinterlassen können (für Norwegen: Gulbrandsen und Langsether 2000). Die Leistungen in die Gegenrichtung, d.h. von den erwachsenen Kindern an die Eltern, sind seltener materieller Art; es handelt sich vielmehr häufig um Pflegeleistungen, wenn die Eltern gebrechlich werden, und um Arbeitsleistungen in und um das Haus. Auch diese Pflege- und Unterstützungsleistungen werden komplementär zu den öffentlichen Leistungen der Gemeinde bzw. des Gesundheitswesens erbracht. Auch hier scheint zu gelten, dass eine gute öffentliche Versorgung die Leistungen der erwachsenen Kinder an ihre Eltern nicht reduziert, sondern eher in Gang hält (für Deutsche und türkische Migranten in Deutschland: Lorenz-Meyer und Grotheer 2000). 
Es ist also nicht so, dass die Verteilungsregeln im Independenzmodell keine solidarischen Praktiken zwischen den Generationen vorsehen, wenn die Kinder erst erwachsen geworden sind. Ganz im Gegenteil sind diese sehr intensiv. Sie sind aber von einem sehr spezifischen Charakter. Man kann die wichtigen Punkte so benennen: Erstens fließen die Leistungen, insbesondere die materiellen, vor allem von „oben“ nach „unten“ in der Altersabfolge; das wurde oben schon ausgeführt. Zweitens ist die weltweit verbreitete Koresidenz zwischen Eltern und erwachsenen Kindern zwar auch in Ländern mit diesem Modell nicht ausgeschlossen, sie ist aber eine Lösung, die vor allem bei finanziellen Engpässen gewählt wird und die als dauerhafte eher vermieden wird. Auch die vergleichsweise hohen Raten von Koresidenz in den europäischen Mittelmeerländern sind unter anderem prekären ökonomischen Bedingungen geschuldet (Szydlik 2016). Drittens sind die Transfers nicht durch eine klare informelle oder gar formelle Verpflichtung motiviert, vielmehr hat der Transfer einen hohen emotionalen Gehalt. Es ist ein heikler Tausch von Materiellem und von Emotionen, der geschickt in Szene gesetzt werden muss. Martine Segalen (1990) spricht für Frankreich davon, dass die Emotionalität zwischen den Generationen asymmetrisch sei und die Eltern mit zunehmendem Alter stärker von der Zuneigung der Kinder abhängig seien als umgekehrt. Geschenke, Darlehen und Dienste an die Kinder seien auch ein Mittel, deren Zuneigung zu gewinnen resp. zu erhalten. Beide Seiten durchschauten das, aber es dürfe nicht offen ausgesprochen werden. Diese Einschätzung deckt sich mit einer bereits etwas älteren französischen Untersuchung von Roussel und Bourguignon (1975). Es handelt sich um eine ethnographische Studie zur Praxis größerer Schenkungen der Eltern an die erwachsenen Kinder, vor allem bei einer Eheschließung. Die Autoren resümieren: „Es muss alles so laufen, als ob die Eltern nichts schenken und die Kinder nichts empfangen würden“" (S. 244). Gelegentlich fungieren dann auch die Enkelkinder als Vermittler der Schenkungen. Damit soll vermieden werden, dass Schenker oder Beschenkte beschämt werden. Viertens ist eine gewisse Gewähr gegeben, dass die Transfers den Familienmitgliedern mit dem größten Bedarf zukommen, weil es sich nicht um eine (stereotype) Verpflichtung handelt, sondern um eine situative Unterstützung (Attias-Donfut und Wolff 2000b).

Fehlen beim intergenerationalen Austausch zwischen Eltern und erwachsenen Kindern im Rahmen des Independenzmodells auch eindeutige und verpflichtende Normen, so gibt es doch die Erwartung gegenseitiger Anteilnahme und einer aufrechtzuerhaltenden Bindung. Ihre Einlösung obliegt weitgehend dem Geschick der Eltern: Nichts in diesem Austausch sollte an eine Verpflichtung denken lassen oder für den Empfänger beschämend sein. Idealerweise errät man die Wünsche und Sorgen der anderen, überrascht man sich gegenseitig, wenn auch mit je anderen Leistungen. Die Intensität der Beziehungen zwischen den Generationen dürfte in dieser Alterskonstellation wesentlich davon abhängen, ob dies gelingt. Es ist bemerkenswert, dass die Austauschbeziehungen zwischen den Eltern und ihren bereits erwachsenen Kindern so wenig geregelt sind, umfassen sie doch zeitlich gesehen den längsten Abschnitt des Lebens: vom Auszug der Kinder aus dem Elternhaus bis zum Zeitpunkt, an dem die alten Eltern gebrechlich und hilfsbedürftig werden. Dann erst werden die normativen Vorgaben für die erwachsenen Kinder eindeutiger und verpflichtender, im Sinne einer gewissen Verantwortung für eine ausreichen- 
de Pflege und Unterbringung der Eltern und unter Umständen (nämlich ab einem gewissen Einkommen und im Fall einer stationären Pflege) sogar eines (allerdings bescheidenen) staatlicherseits eingeforderten finanziellen Beitrags (Felder 4, 5 und 6).

Demgegenüber geht die Vorstellung der „filial piety“ mit der normativen Erwartung einher, dass Kinder ihren Eltern ein Leben lang Gehorsam schulden und verpflichtet bleiben. Die Verpflichtung, für die alten Eltern zu sorgen, ist sogar in der Verfassung einiger Staaten festgeschrieben (Kaplan et al. 2002; Woronow 2007; Esengul 2012). Dass in neuerer Zeit einige asiatische Staaten Gesetze erließen, die für zu wenig pflichtbewusste erwachsene Kinder Strafen ausgesetzt oder verschärft haben, weist allerdings darauf hin, dass die Norm der ,filial piety“ nicht (mehr) durchgängig gesichert ist (Chou 2011; Lee und Kwok 2005). Für einige asiatische Staaten gibt es mehrere neuere Studien, die die Beziehungen zwischen erwachsenen Kindern und ihren Eltern genauer untersucht haben, zum Teil aus Sicht der Eltern, zum Teil aus Sicht der erwachsenen Kinder. Stets fragten sie danach, ob die Verpflichtungen der ,filial piety“ noch wahrgenommen werden. Bei diesen Studien handelt es sich um eine in China regelmäßig durchgeführte Erhebung (Gruijters 2016), eine japanische Surveystudie (Koyano 1996), um vergleichende Daten des East Asian Social Survey für Taiwan, Hong Kong und China (Yeh et al. 2013) und für Südkorea (Ko und Chapman 2017). Die Resultate stimmen weitgehend überein: Die Verpflichtungen der ,filial piety“ werden weiterhin in allen diesen Ländern als bindend anerkannt. Der Kontakt zu den Eltern bleibt intensiv, ob man nun in Koresidenz lebt - was immer noch sehr häufig der Fall ist ${ }^{4}$ - oder weiter entfernt wohnt, z. B. aufgrund von Binnenmigration oder Migration ins Ausland. Nach wie vor werden Leistungen der Sorge und der finanziellen Unterstützung für die Eltern erbracht, nicht nur wenn sie hinfällig oder verarmt sind, sondern als den Eltern geschuldete Dankbarkeit. Dennoch ändert sich das normative Muster in der Art seiner Anwendung: Die Beziehungen zwischen Eltern und heranwachsenden bzw. erwachsenen Kindern werden demokratischer und stärker emotional gefasst (Ko und Chapman 2017). Veränderungen ergeben sich auch durch die stärkere Integration der Frauen in den Arbeitsmarkt, die damit für die Pflege der Eltern weniger Zeit haben; das konnte für Südkorea gezeigt werden (Sung 2003; Shin 2003). Es sind aber auch die alten Eltern selbst, die ihre Vorstellungen und Ansprüche verändern. Das ergaben zwei qualitative Studien, die am Beispiel von chinesischen (Cole und Durham 2007) und von indischen Auswanderern (Sharma und Kemp 2012) untersucht haben, wie Eltern, die ihren erwachsenen Kindern in die USA nachgezogen sind, auf vertraute Privilegien und Dominanzansprüche teilweise verzichtet haben, umgekehrt aber auch ihre Fähigkeit zur Selbsthilfe stärker betonten. Diesen Einstellungswandel ermöglichte ihnen auch die öffentliche Infrastruktur im Einwanderungsland. So berichtete ein chinesisches Ehepaar, wie zuverlässig der Notruf 911 sei und wie gut man dort chinesisch spreche. Da die Kinder sowieso keine Zeit hätten, ihnen Tee zuzubereiten, hätten sie beschlossen, eine eigene Wohnung zu nehmen (Cole und Durham 2007).

\footnotetext{
${ }^{4}$ Kolland (2005) hat anhand von Daten aus asiatischen und afrikanischen Ländern gezeigt, dass die Zahlen zwar leicht zurückgehen, aber immer noch 60-90\% der älteren Menschen mit ihren erwachsenen Kindern zusammenleben.
} 
In diesen Studien wird dennoch stets erkennbar, wie die Eltern ihre Realität und ihre Wünsche weiterhin am normativen Muster der ,filial piety“ messen und daran, wie sie dessen Umsetzung noch bei ihren eigenen Eltern erlebt haben. Sharma und Kemp sprechen in diesem Zusammenhang von ,heuristic devices“ (2012, S. 132), die die Vorstellungen der „filial piety“ für die Beteiligten weiterhin abgäben.

Auch für andere Weltregionen gibt es Hinweise und Belege zu den erheblichen Transferleistungen von erwachsenen Kindern an ihre Eltern. So zeigt eine ethnographische Studie zu einer armen Region in Mexiko, dass die armen elterlichen Haushalte vorübergehend wohlhabend werden konnten, wenn erst einmal genügend Kinder der Familie alt genug geworden waren, um in den USA zu arbeiten und Geld nach Hause zu schicken (Magazine und Ramirez Sanchez 2007). Die Weltbank führt eine Datenbank, die Auskunft gibt über die Geldrücksendungen in verschiedene Länder (World Bank 2020). ${ }^{5}$ Die Gesamtsumme dieser Rücksendungen ist in den Jahren 2017 und 2018 stark gestiegen. 2018 führte das winzige Land Tonga die Liste an mit mehr als $40 \%$ des Bruttosozialproduktes, das durch solche Rücksendungen erbracht wurde, gefolgt von Kirgisistan mit 33\%. Die Geldrücksendungen erfolgen oft an die zurückgelassenen Kinder oder an die eigenen Eltern resp. an andere ältere Verwandte, die zum Teil auch die Betreuung der Kinder übernommen haben, was ebenfalls eine Veränderung im traditionellen Muster der „filial piety“ darstellt. Damit kommen die Rücksendungen auch den alten Angehörigen zugute. Ein großer Teil dieser Rücksendungen dürfte also mittelbar oder unmittelbar dem Gebot der (zum Teil auch modifizierten) „filial piety“ gehorchen. Wer immer der konkrete Nutznießer sein dürfte, es handelt sich um Leistungen generationaler Solidarität, deren wirtschaftliche Bedeutung für die betreffenden Länder und Familien erheblich ist.

Sozialstaatliche Strukturen sind mittlerweile auch in manchen jener Staaten im Entstehen, in denen sich die Transferleistungen zwischen den Altersgruppen weiterhin vornehmlich am Interdependenzmodell orientieren (Kim 2008; Leisering 2011). So werden zunehmend staatliche, betriebliche oder marktwirtschaftliche Systeme der Alterssicherung aufgebaut. Gegen Armut im Alter sichern sie zurzeit jedoch immer noch ungenügend $\mathrm{ab}$. Das hat mehrere Gründe: Zum einen sind viele Leute überhaupt nicht in das Sozialversicherungssystem einbezogen, weil sie schon zu alt waren, als die Altersversicherung eingeführt wurde, oder weil sie in informellen Beschäftigungsverhältnissen ohne Rentenabsicherung arbeiteten. Zum anderen besteht die Altersversicherung zum Teil lediglich in der Zahlung eines recht niedrigen und schnell aufgebrauchten Pauschalbetrages. Es gibt Staaten, wie z.B. Singapur, die sich explizit an dem Grundsatz orientieren, dass die Alterssicherung nur in engen Grenzen eine Staatsaufgabe sei und weiterhin hauptsächlich in den Verantwortungsbereich der Familie oder jedes Einzelnen falle (Williamson et al. 2011). Nur wenige Staaten, in denen die generationalen Verteilungsregeln dem Interdependenzmodell zugeordnet werden können - hier wird z. B. Brasilien genannt (LloydSherlock 2011) -, bieten befriedigende Lösungen im Hinblick auf Alterspensio-

\footnotetext{
5 Als grafisch aufbereiteter Überblick siehe zudem: https://data.worldbank.org/indicator/BX.TRF.PWKR. DT.GD.ZS. Zugegriffen: Febr. 2020.
} 
nen; eine darüber hinausgehende Altenpolitik existiert faktisch aber nicht (Leisering 2011).

Werden von den erwachsenen Kindern zweifellos viele unverzichtbare Leistungen materieller und immaterieller Art für ihre Eltern erbracht, so gibt es doch auch Hinweise dafür, dass die Anforderungen der ,filial piety“ von den jungen Erwachsenen durchaus ambivalent wahrgenommen werden. Dies soll erneut anhand des Beispiels Kirgisistan gezeigt werden. Artikel 37 der Verfassung der Kirgisischen Republik bekräftigt die landeseigenen Traditionen und hält in Absatz 1 fest, dass der Staat das Brauchtum und die Traditionen unterstützen soll, sofern sie nicht den Menschenrechten widersprechen, sowie in Absatz 2, dass der Respekt für die Älteren und die Fürsorge für die Familie und enge Verwandte die Pflicht eines jeden sei. ${ }^{6}$ Die Leistungen, die jüngere Erwachsene den älteren Erwachsenen schulden - einschließlich ihre Unterordnung unter deren Herrschaft -, sollen über den Rahmen von Eltern-KindBeziehungen im engeren Sinne hinausgehen. Das lässt u. a. das in Verfassungsartikel 59 geregelte Recht auf Einrichtung der sogenannten „,courts of aksakaly“ (,Gericht der Weißbärte“) erkennen. Dabei handelt es sich um eine Versammlung der ältesten Einwohner einer Gemeinde, die gerade auch in Familienangelegenheiten Entscheidungen treffen kann. Diese fielen oft zuungunsten der Ansprüche von Frauen aus, wird argumentiert (Manuel 2015); mitunter seien die traditionsorientierten Urteile dieser Gerichte sogar menschenrechtswidrig (Handrahan 2001). Trotz der Respektsbekundungen junger Kirgisinnen und Kirgisen gegenüber ihren Eltern, sind viele von ihnen wenig begeistert, wenn es darum geht, den damit verbundenen weitreichenden Verpflichtungen nachzukommen. So wird z.B. erwartet, dass der jüngste Sohn mit seiner Frau im Haushalt der Eltern lebt und die Schwiegertochter den Löwenanteil an der Hausarbeit bewältigt. In einer großen Umfrage erklärten jedoch über zwei Drittel der befragten jungen Leute, dass junge Paare in einem eigenen Haushalt leben sollten (UNDP 2010, S. 44). Eine Anekdote aus der eigenen Forschungsarbeit vor Ort ist in dieser Hinsicht sehr aufschlussreich: Die Universitätsstudentinnen, die unser Forschungsteam gelegentlich in die Feldforschung mit einbezog oder unterrichtete, erzählten, wie sie beim ersten Kennenlernen von jungen Männern sehr vorsichtig seien. Lerne man einen jungen Mann kennen, sei es angeraten, schnell herauszufinden, ob er jüngere Brüder habe, und falls nicht, auf Distanz zu gehen, denn man wolle ja nicht der Schwiegermutter dienen. Über $60 \%$ aller Haushalte in Kirgisistan bestehen aus Kernfamilien (ebd.). Da es - bei einer durchschnittlichen Geburtenrate von mehr als drei Kindern pro Frau - fast immer mehrere Geschwister gibt, die mit den alten Eltern leben könnten, kann man schlussfolgern, dass die alten Ehepaare fast nie allein leben. Schwittek (2017) konnte in einer qualitativen Studie zu jungen Erwachsenen zeigen, welche heiklen und oft erfolglosen Verhandlungen diese mit den Eltern und den Älteren um ein unabhängigeres Leben führen (Felder 10, 11 und 12).

\footnotetext{
6 „Constitution of the Kyrgyz Republic“, in der geänderten Fassung von 2010, http://www.wipo.int/ wipolex/en/text.jsp?file_id=254747. Zugegriffen: Aug. 2019. Die Betonung der Tradition und der „,filial piety“ ist ein Element des erstarkenden Nationalismus nach dem Ende der Sowjetunion.
} 


\section{Benachteiligte und Begünstigte und das Zusammenspiel von öffentlichem Interesse und Familie}

Die generationale Solidarität regelt einen dichten Fluss an Leistungen zwischen den Gesellschaftsmitgliedern. Die Unmöglichkeit oder der Unwille, im einzelnen Fall die Leistung zu erbringen, sind dabei sicher nicht selten. Beachtung verdienen vor allem aber die systematischen Ungleichheiten, die gerade auch mit der Befolgung der generationalen Verteilungsregeln verbunden sind. Auf der Hand liegt der Zusammenhang mit der sozialen Schichtung: Wohlhabendere Eltern können ihren Kindern mehr zukommen lassen; das gilt auch beim Erbe. In westlichen Gesellschaften findet das zurzeit vermehrt Beachtung: Der generationale Transfer wird als Motor für die Verstärkung sozialer Ungleichheit thematisiert (z. B. Szydlik 2016, S. 165). Hier soll nun aber nicht der Zusammenhang zur sozialen Stratifikation in den Blick genommen werden, sondern die prinzipielle Benachteiligung bestimmter Gruppen, die den generationalen Verteilungsregeln inhärent ist.

Obwohl die Austauschleistungen über den Lebenslauf hinweg für die meisten eine gewisse Balance zwischen Begünstigungen und Benachteiligungen aufweisen, so sind doch auch systematische Benachteiligungen auszumachen, die nicht nur eine frühere Bevorteilung ausgleichen oder in einer späteren Phase wieder kompensiert werden. Mit Blick auf die beiden Grundmodelle und die Frage nach den darin virulenten systematischen Ungleichheiten lassen sich drei allgemeine Thesen aufstellen:

1. Das Zusammenspiel von Altersgruppenzugehörigkeit und Geschlecht schafft besondere, je nach Verteilungsregeln andere Benachteiligungen, von denen überhäufig Frauen und Mädchen betroffen sind.

2. Es ergeben sich in beiden Modellen spezifische Benachteiligungen durch die Dominanz von patriarchalen Familienmustern: Zum einen haben diejenigen mit Nachteilen zu rechnen, die keiner solchen als legitim erachteten Familienform angehören, zum anderen ergeben sich aber auch für bestimmte Angehörige dieser Familien systematisch Nachteile oder Vorteile entsprechend den Interessen und Machtkonstellationen in diesen Familien.

3. Rekonstruiert man das Zustandekommen der Verteilungsregeln, zeigt sich, dass bei ihrer Ausgestaltung und Festschreibung der Staat und Experten in einem hohen Grade involviert waren und bleiben. Die Logik patriarchaler Familien wurde in Regelungen übersetzt, bei denen öffentliche und private Anliegen Hand in Hand gingen.

Diese drei allgemeinen Thesen sollen nun exemplarisch für die unterschiedlichen gesellschaftlichen und generationalen Konstellationen belegt werden. Der Blick auf relative Benachteiligungen und Begünstigungen fokussiert dabei exemplarisch und kontrastierend auf zwei Länder: auf Deutschland für das Independenzmodell und Kirgisistan für das Interdependenzmodell.

Folgen die Verteilungen einem Independenzmodell, so sind die Kinder - wie bereits in Abschnitt 2.1 dargestellt - hinsichtlich der materiellen und immateriellen Leistungen, die ihnen zugutekommen, in der Regel nicht benachteiligt, sondern begünstigt, wenngleich sie natürlich von manchen Anlässen ausgeschlossen sind und in geringerem Maße als Erwachsene über ihre Familiensituation entscheiden 
können. Letzteres bildet z.B. bei der sogenannten „Hochstrittigkeit“ von Familien (Walper et al. 2013) ein großes Problem: Wo Erwachsene eine innerfamiliäre Konfliktsituation auch durch eine Scheidung respektive durch einen Wechsel der Wohnung lösen können, verfügen Kinder nicht über diese Möglichkeit. Auch sind Kinder eine Gruppe, die insbesondere in ihren Familien mehr Gewalt ausgesetzt ist (Albert und Bühler-Niederberger 2015). Eine solche mangelnde Berücksichtigung von Kinderrechten ist aber weltweit gegeben. Eine erhebliche Benachteiligung ist zudem für viele jener Kinder gegeben, die in Familien leben, in denen kein männlicher (Haupt-)Ernährer lebt. Die Armutsziffern bei Kindern Alleinerziehender sind bekannt: Die Absenz eines männlichen Ernährers ist der größte Risikofaktor für Armut. Jedes zweite Kind in Deutschland lebt in einem Haushalt, der von relativer Einkommensarmut betroffen ist; bei Kindern in Paarfamilien ist es nur jedes fünfte Kind (BMAS 2017, S. 257 f.). Zwar sind die Zahlen zur Kinderarmut, gemessen als relative und bedarfsgewichtete Einkommensarmut, wenig verlässlich und schwanken um mehrere Prozentpunkte, je nach Art der Messung (z.B. EU-SILC oder SOEP). Dass aber Kinder Alleinerziehender (überwiegend sind es Frauen) deutlich häufiger in einkommensarmen Haushalten aufwachsen, ist unstrittig (Bühler-Niederberger 2020a, S. 54 ff.). Hier erschöpft sich schnell die Solidarität von Seiten der Erwachsenen mit ihren Kindern. Die Zahlungsmoral und sicher auch zum Teil die Zahlungsfähigkeit von geschiedenen und unverheirateten Vätern ist mangelhaft; zwischen einem Drittel und der Hälfte der unterhaltspflichtigen Väter kommen dieser Verpflichtung nicht nach (Bröckel und Andreß 2015; Leopold 2018).

Die systematische Benachteiligung, die sich für Eltern von minderjährigen Kindern hinsichtlich der von ihnen erwarteten Leistungen im Rahmen des Independenzmodells ergibt, erwächst aus dem Zusammenspiel von mittlerer generationaler Kategorie und Geschlecht. Das gilt in beide Richtungen: für den Transfer zu den Kindern und für den zu den eigenen Eltern oder denen des Ehepartners. Sie betrifft die Frauen: Sie übernehmen den Hauptanteil an der Erziehungs- und Hausarbeit (Dechant et al. 2014). Zeitbudgeterhebungen in Deutschland, die von den Statistischen Ämtern des Bundes und der Länder bislang dreimal durchgeführt wurden, zeigen: Bereits bei Paaren ohne Kinder ist die Verteilung der Hausarbeit ungleich, bei Eltern sind aber die Unterschiede deutlich größer. Im Vergleich zu Paaren ohne Kinder steigern Männer in Familien mit Kindern ihre Beteiligung an Arbeiten für Haushalt und Familie um durchschnittlich täglich zwanzig Minuten auf zwei Stunden 56 min pro Tag; die Frauen steigern sie um zwei Stunden, auf fünf Stunden und $37 \mathrm{~min}$ pro Tag. Die relativen Veränderungen in der Hausarbeitszeit von Männern und Frauen seit den 1990er-Jahren bewegen sich lediglich im Minutenbereich (Klünder und Meier-Gräwe 2017). Mehr Zeit investieren die Frauen später auch für die Betreuung der Enkel (Attias-Donfut und Segalen 2002) und in die Pflegeleistungen für die alternden Eltern. Letzteres tun sie umso mehr, wenn sie verheiratet sind, aber auch als Nichtverheiratete übernehmen sie öfter als Männer die Pflege von Angehörigen (Attias-Donfut und Wolff 2000a). Hinzu kommt ein beträchtlicher Gehaltsunterschied zwischen berufstätigen Frauen und Männern. In Deutschland ist er einer der höchsten Europas (Eurostat 2018). Aus dem bereits Gesagten wird hinreichend klar, dass auch die Renten von Frauen tiefer liegen als die der Männer, also auch ältere Frauen benachteiligt werden. Hohe Rentenanwartschaften von über 
$1500 €$ im Monat erreichten im Jahr 2017 in den alten Bundesländern nahezu ausschließlich Männer, nämlich $22 \%$ von ihnen, während der Anteil bei den Frauen bei lediglich $4 \%$ lag; in den neuen Bundesländern lagen die entsprechenden Anteile aufgrund der ganz unterschiedlichen Erwerbsbiografien bei ungefähr 9,5\% bzw. 10\% (Deutsche Rentenversicherung 2019, S. 94).

Fragt man nun nach der Rolle des Staates bei der Etablierung der Verteilungsregeln - und das heißt also auch ihres patriarchalen Bias -, so kann man für das Independenzmodell auf viele historische Arbeiten zurückgreifen, die den Einfluss des Staates und seiner Gesetzgebung deutlich erkennen lassen. In den Verteilungsregeln, die diesem Modell folgen, zeigt sich der Entwurf des Kindes als „,economically useless, but emotionally priceless“, wie ihn Zelizer (1985, S. 57) zuerst für die städtische bürgerliche Mittelschicht in den USA in der zweiten Hälfte des 19. Jahrhunderts beschrieben hat. In den westeuropäischen Ländern und den USA wurde dieser Anspruch zunächst von Experten und von Sozialreformern und schließlich vom Staat erhoben und seit dem Übergang zum 20. Jahrhundert schrittweise in allen sozialen Schichten durchgesetzt. Die Durchsetzung des bürgerlichen Modells des männlichen Alleinverdieners und Familienernährers, das in vielen westlichen Ländern und vor allem auch in Deutschland fast für das ganze 20. Jahrhundert zum ausschließlich akzeptierten wurde, führte ja nicht nur dazu, dass die Frauen verstärkt von der Erwerbsarbeit ausgeschlossen wurden, sondern beinhaltete auch einen Ausschluss der Kinder aus der Arbeitswelt. Beide Entwicklungen setzten ungefähr zur selben Zeit ein und wurden mit einem Bündel aus disziplinierenden Maßnahmen und sozialpolitischen Anreizen sukzessive durchgesetzt. Neben dem Verbot der Kinderarbeit umfassten diese Maßnahmen u.a. die Einführung von Vormundschaft und Fürsorgeerziehung, eine rigorose Inpflichtnahme der Frauen für ihre Aufgaben als Mutter, einen sozialen Wohnungsbau, der bestimmte Familienstrukturen schon rein räumlich erzwang, und die Schulpflicht und ihre Überwachung (Donzelot 1980; Badinter 1984; Mahood 1995; Hendrick 1997). Staat und Experten versprachen sich von der Familie mit einer strikten Geschlechter- und Generationenordnung auch ein Mehr an gesellschaftlicher Ordnung: die Einbindung eines jeden in einen autoritären und gleichzeitig emotional dichten Zusammenhang - als Mann, Frau, Kind. Soweit es die Kinder betraf, setzte man auch auf die Internalisierung der gesellschaftlichen Normen (im Sinne des Aufbaus eines Über-Ichs) durch die besondere kleinfamiliäre Struktur (Donzelot 1980; Schlumbohm 1983; diese ordnende Funktion sprechen auch noch Parsons und Bales [1955] der Familie zu). Zudem stand das nun ökonomisch ,wertlose“ Kind in ganz anderer Weise für die Einrichtungen der Bildung und deren gesellschaftlichen Ordnungsauftrag zur Verfügung, nicht nur zeitlich, sondern auch aufgrund seiner nun ganz und gar abhängigen Position (De Coninck-Smith 1997). ${ }^{7}$

Eine solche Geschlechter- und Generationenordnung wurde nicht nur in Deutschland, sondern in fast allen Ländern, die man dem Independenzmodell zuordnen kann, etabliert. Für Westdeutschland ist aber bemerkenswert, dass die Angst, die Aufwei-

\footnotetext{
${ }^{7}$ Der Herauslösung von Kindern und den sie umsorgenden Müttern aus den erwerbswirtschaftlichen Zusammenhängen lagen gleichwohl auch handfeste ökonomische Motive zugrunde: der Aufbau von Humankapital, das später dem Arbeitsmarkt zur Verfügung gestellt werden kann.
} 
chung dieser Ordnung könnte zu Unordnung führen, noch bis in die 1980er-Jahre die Politik umtrieb. Noch zu dieser Zeit wurden besorgte Debatten über die Verwahrlosung der Kinder berufstätiger Frauen, der sogenannten ,Schlüsselkinder“ geführt - auch unter wissenschaftlicher Beteiligung (Sommerkorn 1988). Vor allem ist es die Familienpolitik der Nachkriegszeit, über die die patriarchalen Familienstrukturen konserviert wurden. Damals wurden die familienpolitischen Richtlinien und Leistungen festgelegt, die im Wesentlichen bis heute gelten. Über Steuervergünstigungen, sozialversicherungsrechtliche Regelungen und Zuschläge für verheiratete Beamte privilegieren diese die Ehe, insbesondere die Ehe, in der ein Partner wesentlich mehr verdient als der andere - und zumeist ist das immer noch der Mann. Bei ihrer Einführung wurden diese familienpolitischen Maßnahmen im Parlament und den öffentlichen Debatten explizit mit dem Interesse des Staates an patriarchalen Familienstrukturen begründet. Der erste Familienminister der Nachkriegszeit sprach von den ,rechtschaffenen Kindern“, die in ,innerlich gesunden Familien“ erzogen würden, und stellte sie den „streunenden Kindern“ aus Scheidungsfamilien und Familien mit berufstätigen Müttern gegenüber (Langer-El Sayed 1980, S. 99; Gerlach 1996, S. 189). Mag der Staat an der generellen Verbreitung und Langlebigkeit dieses Musters zwar einen entscheidenden Anteil haben, so hat er es aber nicht erfunden, sondern griff auf die Vorlage zurück, die das Bürgertum geliefert hatte. Mit der patriarchal strukturierten Familie und der Erziehung, die diese leiste, begründete es in Deutschland seit dem ausgehenden 18. Jahrhundert seine Überlegenheit als Stand (Budde 1994, S. 11). Ein Teil der Härten für Kinder und Frauen, die aus dem Zusammenspiel zwischen den Verteilungspraktiken im privaten Raum und den staatlichen Regelungen entstehen bzw. entstehen können, werden allerdings durch den Staat auch abgefedert, z.B. durch einen Unterhaltsvorschuss bei ausbleibenden Unterhaltszahlungen und durch Sozialleistungen wie das Kindergeld. Das geschieht aber ganz offensichtlich nicht in ausreichendem Maße, wie die oben erwähnten aktuellen Kinderarmutszahlen belegen. Die Begünstigung der Familien, die dem männlichen Alleinverdienermodell entsprechen, wie sie bei höherem Einkommen erheblich ist, wird in Deutschland zwar immer wieder kritisch diskutiert. Seit Jahrzehnten dreht sich diese Debatte etwa um das sogenannte Ehegattensplitting, das außer Deutschland in dieser Weise kein anderes Land praktiziert; verändert oder gar abgeschafft wurde es aber bisher nicht. Eine solche Änderung ist auch in unmittelbarer Zukunft nicht zu erwarten. Es ist vor diesem Hintergrund wenig verwunderlich, dass neuere Maßnahmen, die die Verteilungsregeln gerechter gestalten wollen - so z. B. die in dieser Hinsicht revidierten Bestimmungen des „Elterngelds“ (früher „Erziehungsgeld“) -, nur zögerlich in tatsächlich egalitärer Weise von den Eltern angenommen werden (Destatis 2019).

In Ländern, in denen das Interdependenzmodell vorherrscht, ist die Situation der Kinder in vielen Fällen keine besonders bevorzugte. In Kirgisistan können zwar die Arbeiten, die den Kindern übertragen werden, ihnen auch Anerkennung bringen. Aber die Leistungen werden von ihnen mit einer harschen Disziplin eingefordert, über die die Kinder klagen. Darüber hinaus sehen sich die Kinder mit den hohen Ambitionen und Erwartungen ihrer Eltern konfrontiert, worauf schon in Abschnitt 2.1 hingewiesen wurde (Bühler-Niederberger und Schwittek 2014; Bühler-Niederberger 2020b). Es trifft hier die Kinder weit härter, wenn sie außerhalb einer Ehe auf- 
wachsen, als in Ländern, in denen man sich an den Regeln des Independenzmodells orientiert. Man könnte glauben, dass in Anbetracht der Koresidenz und also auch von größeren Haushalten sich die Situation von Kindern alleinerziehender Mütter von derjenigen verheirateter Mütter weniger unterscheidet als in westlichen Ländern. Aufgrund der gängigen virilokalen Koresidenz stehen jedoch viele Frauen und ihre Kinder nach einer Scheidung zunächst auf der Straße. Die Benachteiligung bestimmter Kinder im Familienverband ist aber nicht nur eine wirtschaftliche, sie betrifft auch den Wert des Kindes. Dieser resultiert weitgehend aus dem Wert seines Vaters resp. der Vaterlinie. In Kirgisistan werden Kinder, die in Haushalten der Vaterlinie aufwachsen - seien das die Eltern, die Brüder oder der Onkel des Vaters -, als „Enkel“ bezeichnet; Kinder, die in der Mutterlinie aufwachsen, dagegen als „Nichten“ und „Neffen“. Letztere haben einen klar geringeren Wert und geringere Ansprüche gegenüber der Verwandtschaft. ${ }^{8}$ Die angeblich wachsende Zahl von „Neffen“ und „Nichten“ - Statistiken dazu existieren nicht - tauchte vor einigen Jahren als soziales Problem in den öffentlichen Debatten auf. Der Staat versucht zwar, die Situation von Kindern und Frauen nach einer Scheidung zu verbessern; so sieht er z.B. hohe Unterhaltszahlungen durch die Väter und eine drakonische Bestrafung ihrer Nichtbegleichung vor. Die Regelungen werden aber nur selten durchgesetzt. Ein stark informeller Arbeitsmarkt macht es zum Beispiel schwer, die Höhe der Einkommen geschiedener Männer genau festzustellen. Offen ist auch, wieweit die staatlichen Gesetze in den ländlichen Regionen durchzusetzen sind bzw. dies von den dort zuständigen Instanzen überhaupt versucht wird.

Als Benachteiligte können auch in Anbetracht der von ihnen erwarteten „filial piety" die erwachsenen Kinder in Kirgisistan gesehen werden: Sie schulden den Eltern Gehorsam, sie können ihren Wohnort in vielen Fällen nicht selber wählen, genauso wenig wie die Studienrichtung, den Beruf oder den Heiratspartner; das zeigen Jugend-Surveys (Esengul 2012). Besonders groß ist die Benachteiligung für die jungen Frauen. Sie werden - wie bereits ausgeführt - nicht selten zu Dienstmädchen der Schwiegermütter. Die mediale Vermarktung dieser Praxis in der Reality-GameShow des kirgisischen staatlichen Fernsehens „Wie gefallen Sie Ihrer Schwiegermutter" sorgte dementsprechend für politischen Aufruhr. In dieser Show kämpften zehn junge Frauen - darunter Studentinnen und Beamtinnen - um den Titel der besten Schwiegertochter in spe: beim Servieren von Tee, Backen von Brot, Waschen und Putzen. ${ }^{9}$ Dies führte zu einer Petition an die Politik durch aufgebrachte feministische Organisationen. Immerhin ist dies ein Hinweis darauf, dass die autoritärpaternalistischen Bestandteile der "filial piety“ nicht mehr von allen bedingungslos akzeptiert werden.

Ergeben sich im Rahmen des Interdependenzmodells besonders starke Benachteiligungen für jüngere Frauen, so ist die Situation von älteren Frauen aufgrund der

\footnotetext{
${ }^{8}$ Der Roman Der weiße Dampfer des kirgisischen Autors und Nobelpreisträgers Tschingis Aitmatow ist die traurige Geschichte eines Kindes, das bei seinen Verwandten mütterlicherseits lebt - geduldet, aber nicht geschätzt.

${ }^{9}$ Bei YouTube eingestellte Sendungsausschnitte zeigen, wie sie dabei von der von einer Schauspielerin gespielten virtuellen Schwiegermutter kontrolliert und auch heruntergemacht werden. https://www.youtube. com/watch?v=qR_nZe1z31A. Zugegriffen: Febr. 2020.
} 
in diesem Modell vorherrschenden normativen Muster relativ privilegiert. Haben sie Söhne großgezogen, verfügen sie über ein hohes Ansehen, außerdem über eine Schwiegertochter als Magd, die sie sich oft selbst ausgewählt haben, indem sie die Eheschließung der Söhne arrangierten. Mithin verfügen sie über eine besondere Macht in ihrem Haus. Finanziell sind sie prinzipiell nicht schlechter gestellt als die Männer ihres Alters, da die erwirtschafteten Altersrenten ohnehin nur einen Bruchteil der Versorgung sicherstellen und das Meiste von den Nachkommen erbracht wird.

Zum Zusammenwirken von Staat und Familieninteressen in Kirgisistan liegen nur Erkenntnisse für die jüngste Geschichte vor. Lange dürfte die Regelung solcher Angelegenheiten den Familienverbänden überlassen gewesen sein. Zur Sowjetzeit bemühte sich der Staat um eine Besserstellung der Frauen. Soweit es die Bildung und die Partizipation am Arbeitsmarkt betraf, scheint diese auch gelungen zu sein (Pascall und Manning 2000; Constantine 2007). Daneben existieren aber bis heute traditionelle Heiratspraktiken, die die Frauen benachteiligen. $\mathrm{Zu}$ nennen sind hier die Verheiratung Minderjähriger, die Entführung junger Frauen (um sie zur Hochzeit zu zwingen $)^{10}$ oder die religiöse Eheschließung, die eigentlich nicht legal ist, aber bei deren Auflösung die Frauen auch nicht wirklich auf staatliche Hilfe setzen können. Zwar sind diese Praktiken verboten, und auf sie werden sogar hohe Strafen ausgesetzt; es wird aber von den Behörden nicht mit erkennbarem Einsatz und noch weniger mit Erfolg dagegen vorgegangen (Kim 2020). Man trifft in der kirgisischen Gesellschaft allenthalben auf eigenartige Widersprüche als Folge der öffentlichen (Teil-)Emanzipation der Frauen, die die Sowjetzeit leistete, und der fortbestehenden traditionellen Privatheitsmuster: So berichtet Jessica Schwittek (2017, S. 9 ff.) in ihrer Studie über junge Erwachsene in Kirgisistan von einer Dekanin und an ihrer Habilitation arbeitenden Naturwissenschaftlerin, die darüber klagt, dass sie vor einigen Jahren gekidnappt und zur Heirat gezwungen wurde, dagegen aber nichts unternommen hat, sondern in der Ehe ausharrt.

Zweifellos ist der kirgisische Staat in einem geringeren Maße bei der Etablierung der Familienmuster involviert als der deutsche Staat dies war und ist. Er unterstützt die traditionalen patriarchalen Muster teilweise durch Untätigkeit und teilweise durch das Bekenntnis zum normativen Muster der ,filial piety“, gerade auch in dessen autoritären Gehalt. Von dieser Bekräftigung der ,filial piety“ kann sich der Staat Entlastung bei der Sorge für die alten Menschen versprechen; er kann sich davon aber auch insgesamt eine höhere Akzeptanz von Autorität als Herrschaftsmodus erhoffen.

\section{Fazit - Ordnungen des Privaten: Leistungen, Grenzen, Gestaltbarkeit}

In diesem Beitrag ging es darum, die These zu belegen, dass Leistungen der Unterstützung und Umverteilung zwischen den Gesellschaftsmitgliedern in einem hohen

\footnotetext{
${ }^{10}$ Die Häufigkeit des sogenannten Brautraubes ist umstritten; man findet Schätzungen, dass zwischen 20 und 50\% aller Eheschließungen so zustande kämen. Die wirkliche Anzahl ist schwer zu ermitteln, weil die Frauen in der Regel auf eine Anzeige verzichten (Molchanova und Galako 2017).
} 
Maß entlang des sozial definierten Alters organisiert werden. Trotz der Orientierung an diesem quasi-natürlichen Merkmal können die konkreten Verteilungsregeln und -praktiken und deren normative Grundlagen sehr unterschiedlich ausfallen. Dies sollte die am Anfang des Beitrages entwickelte Heuristik der typologischen Unterscheidung von zwei nahezu konträren Modellen des generationalen Austausches zwischen Eltern und Kindern verdeutlichen, die hier als Independenz- und als Interdependenzmodell bezeichnet werden. Beide Modelle spiegeln die jeweiligen Grundregeln und normativen Grundmuster wider. Diese umspannen den ganzen Lebenslauf in einem Gesamtaufbau, der in sich je stimmig, ja weitgehend zwingend ist. Um ein Beispiel zu nennen: Das normative Muster der ,guten Kindheit“ mit seinem Individualisierungsprogramm resultiert nicht zufällig in einem spezifischen Erwachsenenstatus, der es den Erwachsenen als persönliche Entscheidung überlässt, wie sie die Beziehungen zu ihrer Herkunftsfamilie gestalten. Eine solche Kindheit würde schlecht zu Verpflichtungen der erwachsenen Kinder passen, in Koresidenz mit den Eltern zu leben und Gehorsam gegenüber älteren Familienangehörigen zu zeigen. Und doch gibt es auch in diesem Modell eine Reihe von Verpflichtungen, denen dann freiwillig nachzukommen allgemein erwartet wird. Ob die konkreten Regeln und Praktiken der Verteilung von Unterstützungsleistungen zwischen den Generationen aber dem einen oder dem anderen Modell entsprechen, sie sichern wechselseitige Leistungen von eindrücklichem Umfang ab.

Beide Verteilungsmuster haben ihre eigene, lange Geschichte; zumindest Bestandteile solcher generationalen Ordnungen existieren seit Jahrhunderten in den Regionen, in denen sie noch heute gelten. So sind Mehrgenerationenhaushalte bereits im frühneuzeitlichen West- und Mitteleuropa, ja sogar schon im Mittelalter eher die Ausnahme als die Regel (Goody 1989; Segalen 1990). Die Eheschließung erfolgte im Allgemeinen recht spät und war zumeist mit einer eigenen Hausstandsgründung verbunden. Man spricht in diesem Zusammenhang vom „European marriage pattern“ (Hajnal 1965). Es bedeutete, dass in Europa von jeher kaum Mittel innerhalb der Familie von der jüngeren an die ältere Generation flossen. Eine noch längere Geschichte wird den Verpflichtungen der ,filial piety“ zugeschrieben, die für den asiatischen Raum auf die Lehren von Konfuzius zurückgeführt werden.

Welchem Muster aber die Verteilungsregeln auch immer entsprechen, sie können in Grenzen den Bedingungen der Gegenwart angepasst werden; sie haben also in diesem Sinne auch eine Zukunft. Die Verteilungspraktiken im Rahmen des Independenzmodells reagieren zum Beispiel flexibel auf den veränderten und verlängerten Übergang ins wirtschaftlich selbstständige Erwachsenenalter durch lange Ausbildungszeiten und prekäre Anstellungsverhältnisse. Das Ausmaß und die Art der Zuwendungen an erwachsene Kinder von Seiten ihrer Eltern lassen auch erkennen, dass durchaus ein Bewusstsein für die Problematik eines solchen Austausches und seinen potenziell beschämenden Charakter besteht. Ebenso sind die Regeln und Praktiken, die dem Interdependenzmodell zugerechnet werden können, durchaus anpassungsfähig, wenn es darum geht, mit der Art von Migration umzugehen, wie sie sich in den vergangenen Jahrzehnten im globalen Maßstab entwickelt hat, bei der viele Kinder von ihren Eltern im Heimatland zurückgelassen werden (müssen). Die in ihrer zeitlichen Dauer ungewissen Transmigrations- und Binnenwanderungsprozesse stellen diese Familien vor besondere Probleme; hier erleichtert die Praxis der Ko- 
residenz die Versorgung der zurückgelassenen Kinder durch Großeltern und andere Verwandte. Umgekehrt steigen auf diese Weise auch die finanziellen Leistungen von Seiten der erwachsenen Kinder an ihre Eltern in Form von Geldrücksendungen von Arbeitsmigranten. Die heranwachsenden Kinder wiederum übernehmen einen Teil jener Arbeitsleistungen für die älteste Generation, die ihren Eltern zugedacht waren (Hu 2018).

Dass die Anpassung an die aktuellen Bedingungen ihre Grenzen hat, zeigte der Blick auf die Benachteiligten, die es in beiden Fällen gibt. So wurde auf die Benachteiligung von Kindern alleinerziehender Eltern hingewiesen, die durch die familienpolitische Bevorzugung der männlichen Ernährerfamilie zustande kommt, da wo die Regeln des Independenzmodells leitend sind. Ebenso ist die starke Inanspruchnahme der Frauen für das intensiv fördernde Kindheitsmuster schwer vereinbar mit einer dauerhaften Präsenz auf dem Arbeitsmarkt, die ihnen erst ein Einkommen und eine hinreichende Altersversorgung sichert. Hier können allerdings die nationalen wohlfahrtsstaatlichen Lösungen für beide Probleme sehr unterschiedlich ausfallen. Skevik (2003) zeigt in einem Vergleich der wohlfahrtsstaatlichen Regelungen in Norwegen und England für Kinder, die bei einem Elternteil leben, wie sehr die norwegische Politik eher an den individuellen Bedarfslagen der Kinder orientiert ist und die englische dagegen traditionell an der Norm und dem Wert einer vollständigen Familie festhält. Ebenso zeigen sich erhebliche Unterschiede im Ausmaß, in dem die Staaten durch sozialpolitische Maßnahmen eine Arbeitsmarktpartizipation von Frauen trotz intensiv fördernder Kindheitsmuster erlauben oder sogar aktiv fördern.

Folgen die Verteilungsregeln dem Interdependenzmodell, kann eine Scheidung bzw. die Außerehelichkeit von Kindern ein noch weit größeres Problem für die Frauen und Kinder darstellen. Demgegenüber scheint die Präsenz der Frauen auf dem Arbeitsmarkt weniger problematisch, auch weil die Erwartungen an die Beiträge der Kinder hier andere sind, gesetzt den Fall, es sind überhaupt genügend bezahlte Arbeitsmöglichkeiten im Land vorhanden. Die Norm der „filial piety“ ist nicht selten mit dem Problem einer asymmetrischen Autoritätsbeziehung zwischen den Generationen behaftet. Die Demokratisierung der Beziehungen zwischen den erwachsenen Kindern und den alten Eltern scheint in manchen Ländern durchaus zu gelingen - das zeigten mehrere Studien. Im Falle Kirgisistans gelingt sie bislang nur ungenügend und erzeugt ambivalente Haltungen bei den jungen Leuten. Auch die Alterssicherung bleibt in vielen Ländern stark ungenügend.

Die partielle bzw. sukzessive Anpassung der Verteilungsregeln an aktuelle Bedingungen lässt die Vermutung zu, dass es auch zu einer Angleichung der Verteilungsregeln der beiden Muster kommen kann. In einigen der oben referierten Beispiele deutet sich eine solche Annäherung an: wenn etwa in westlichen Ländern junge Erwachsene aufgrund des schwierigen Einstieges in den Arbeitsmarkt bei den Eltern wohnen bleiben oder wieder einziehen oder wenn chinesische und indische Eltern in den USA ihre Selbstständigkeit betonen, weil die erwachsenen Kinder doch keine Zeit für sie haben. Aber trotz solcher einzelnen Abweichungen scheinen die jeweiligen Muster für die Beteiligten einen Orientierungsrahmen abzugeben, der als Alltagsheuristik bezeichnet wurde.

Internationale Entwicklungsorganisationen - allen voran die Weltbank, die sich als Vordenker der globalen Entwicklungspolitik versteht - treten allerdings immer 
wieder mit Vorschlägen und Programmen hervor, die auf eine Angleichung der Muster zielen. So haben sie für den Bereich der Kindheit, den sie sehr stark fokussieren, ein ,one size fits all“"-Ideal entwickelt (Schaub et al. 2017): eine intensiv und früh geförderte Kindheit, die einzig im Dienst der Bildung steht und auf Individualisierung ausgerichtet ist. Die Weltbank deklariert denn auch ihre „Education Strategy 2020“ wie folgt: „Invest early. Invest smartly. Invest for all.“ (World Bank 2011, S. 4) Gleichzeitig hat sie den Bereich der Alterspolitik mehr als stiefmütterlich behandelt und vor hohen staatlichen Leistungen und Umlagesystemen gewarnt, die angesichts der demografischen Entwicklung untragbar und marktschädigend seien (World Bank 1994), eine Sichtweise, die in dieser Radikalität später etwas zurückgenommen wurde (Holzmann und Hinz 2005). Die hier vorgestellte Perspektive eines lebenslaufumspannenden Solidaritätsgefüges zwischen den Generationen sollte daher auch als ein Hinweis darauf verstanden werden, dass alle Vorschläge zur Sozial- und Entwicklungspolitik nicht einzelne Lebensphasen und Generationen isoliert voneinander betrachten, sondern das gesamte generationale Solidaritätsgefüge einer Gesellschaft in den Blick nehmen sollten. Hier müsste dann die Einsicht Platz greifen, dass dieses Gefüge aus guten Gründen und mit je eigenen Vor- und Nachteilen durchaus unterschiedlich ausfallen kann.

Funding Open Access funding provided by Projekt DEAL.

Open Access Dieser Artikel wird unter der Creative Commons Namensnennung 4.0 International Lizenz veröffentlicht, welche die Nutzung, Vervielfältigung, Bearbeitung, Verbreitung und Wiedergabe in jeglichem Medium und Format erlaubt, sofern Sie den/die ursprünglichen Autor(en) und die Quelle ordnungsgemäß nennen, einen Link zur Creative Commons Lizenz beifügen und angeben, ob Änderungen vorgenommen wurden.

Die in diesem Artikel enthaltenen Bilder und sonstiges Drittmaterial unterliegen ebenfalls der genannten Creative Commons Lizenz, sofern sich aus der Abbildungslegende nichts anderes ergibt. Sofern das betreffende Material nicht unter der genannten Creative Commons Lizenz steht und die betreffende Handlung nicht nach gesetzlichen Vorschriften erlaubt ist, ist für die oben aufgeführten Weiterverwendungen des Materials die Einwilligung des jeweiligen Rechteinhabers einzuholen.

Weitere Details zur Lizenz entnehmen Sie bitte der Lizenzinformation auf http://creativecommons.org/ licenses/by/4.0/deed.de.

\section{Literatur}

Alanen, L. (2001). Childhood as a generational condition: Children's daily lives in a central Finland town. In L. Alanen \& B. Mayall (Hrsg.), Conceptualizing child-adult relations (S. 129-143). London: Falmer.

Alanen, L., \& Mayall, B. (Hrsg.). (2001). Conceptualizing child-adult relations. London: Falmer.

Albert, L., \& Bühler-Niederberger, D. (2015). Invisible children? Professional bricolage in child protection. Children and Youth Services Review, 57, 149-158.

Arber, S., \& Attias-Donfut, C. (Hrsg.). (2000). The myth of generational conflict: The family and state in ageing societies. London: Routledge.

Ariès, P. (1978). Geschichte der Kindheit. München: dtv.

Attias-Donfut, C., \& Lapierre, N. (1997). La Famille Providence. Trois générations en Guadeloupe. Paris: La Documentation Française.

Attias-Donfut, C., \& Segalen, M. (2002). The construction of grandparenthood. Current Sociology, 50, 281-284. 
Attias-Donfut, C., \& Wolff, F.-C. (2000a). Complementarity between private and public transfers. In S. Arber \& C. Attias-Donfut (Hrsg.), The myth of generational conflict: The family and state in ageing societies (S. 47-68). London: Routledge.

Attias-Donfut, C., \& Wolff, F.-C. (2000b). The redistributive effects of generational transfers. In S. Arber \& C. Attias-Donfut (Hrsg.), The myth of generational conflict: The family and state in ageing societies (S. 22-46). London: Routledge.

Badinter, E. (1984). Die Mutterliebe. Geschichte eines Gefühls vom 17. Jahrhundert bis heute. München: dtv.

Bischoff-Pabst, S., \& Knoll, A. (2015). Förderbedürftige Kindheit - Zur Konstruktion eines Kindheitsbildes aus der Sicht von Eltern. Diskurs Kindheits- und Jugendforschung, 10, 415-429.

BMAS (2017). Lebenslagen in Deutschland. Der Fünfte Armuts- und Reichtumsbericht der Bundesregierung. Berlin: Bundesministerium für Arbeit und Soziales.

BMFSFJ (Hrsg.). (2006). Siebter Familienbericht. Familie zwischen Flexibilität und Verlässlichkeit. Perspektiven für eine lebenslaufbezogene Familienpolitik. Berlin: Bundesministerium für Familie, Senioren, Frauen und Jugend. https://www.bmfsfj.de/bmfsfj/service/publikationen/7--familienbericht/ 74010. Zugegriffen: Febr. 2020.

Bray, R., \& Dawes, A. (2016). Parenting, family care and adolescence in East and Southern Africa: An evidence-focused literature review. Florence: UNICEF Office of Research-Innocenti. https://www. unicef-irc.org/publications/pdf/IDP_2016_02.pdf. Zugegriffen: Febr. 2020.

Bröckel, M., \& Andreß, H.-J. (2015). The economic consequences of divorce in Germany: What has changed since the turn of the millennium? Comparative Population Studies, 40, 277-312.

Bruckauf, Z., \& Rees, G. (2017). Children's participation in housework. Innocenti Research Brief 201717. Florence: UNICEF-Innocenti.

Budde, G.-F. (1994). Auf dem Weg ins Bürgerleben. Kindheit und Erziehung in deutschen und englischen Bürgerfamilien 1840-1914. Göttingen: Vandenhoeck \& Ruprecht.

Bühler-Niederberger, D. (2010). Childhood sociology - Defining the state of the art and ensuring reflection. Current Sociology, 58, 155-164.

Bühler-Niederberger, D. (2020a). Lebensphase Kindheit. Theoretische Ansätze, Akteure und Handlungsräume. 2., überarb. Aufl. München: Juventa

Bühler-Niederberger, D. (2020b). Success - a ,leitmotiv“ of Kyrgyz childhoods. In C. Baraldi \& L. Rabello De Castro (Hrsg.), Global childhoods in international perspective (S. 129-145). London: Sage.

Bühler-Niederberger, D. (2020c). Intergenerational solidarities - Toward a structural approach to childhood sociology. In R. Braches (Hrsg.), The future of childhood research. Leverkusen: Verlag Barbara Budrich. (im Erscheinen)

Bühler-Niederberger, D., \& Schwittek, J. (2014). Young children in Kyrgyzstan. Agency in tight hierarchical structures. Childhood, 21, 502-516.

Chou, R. J.-A. (2011). Filial piety by contract? The emergence, implementation, and implications of the „Family Support Agreement“" in China. The Gerontologist, 51, 3-16.

Cole, J., \& Durham, D. (Hrsg.). (2007). Generations and globalization. Bloomington: Indiana University Press.

De Coninck-Smith, N. (1997). The struggle for the child's time - at all times. School and children's work in town and country in Denmark from 1900to the 1960s. In N. de Coninck-Smith, B. Sandin \& E. Schrumpf (Hrsg.), Industrious children. Work and childhood in the Nordic countries 1850-1990 (S. 129-159). Odense: University Press.

Constantine, E. A. (2007). Practical consequences of Soviet policy and ideology for gender in Central Asia and contemporary reversal. In J. Sahadeo \& S. Zanca (Hrsg.), Everyday life in Central Asia: Past and present (S. 115-126). Bloomington: Indiana University Press.

Coppens, A. D., Alcalá, L., Rogoff, B., \& Mejía-Arauz, R. (2018). Children's contribution to family work: Two cultural paradigms. In S. Punch, R. Vanderbeck \& T. Skelton (Hrsg.), Families, intergenerationality, and peer group relations (S. 187-213). Singapore: Springer.

Crivello, G., \& Espinoza-Revollo, P. (2018). Care labour as temporal vulnerability in woman-child relations. In R. Rosen \& K. Twamley (Hrsg.), Feminism and the politics of childhood: Friends or foes? (S. 139-155). London: UCL Press.

Dechant, A., Rost, H., \& Schulz, F. (2014). Die Veränderung der Hausarbeitsteilung in Paarbeziehungen. Zeitschrift für Familienforschung, 26, 144-168.

Destatis (2019). Elterngeld: $4 \%$ mehr Empfängerinnen und Empfänger im Jahr 2018. Pressemitteilung Nr. 145 vom 11. April 2019. https://www.destatis.de/DE/Presse/Pressemitteilungen/2019/04/PD19_ 145_22922.html. Zugegriffen: Apr. 2020. 
Deutsche Rentenversicherung (2019). Versichertenbericht 2019. Statistische Analysen zu den Versicherten der Deutschen Rentenversicherung. https://www.deutsche-rentenversicherung.de/ SharedDocs/Downloads/DE/Statistiken-und-Berichte/Berichte/versichertenbericht_2019.pdf? blob=publicationFile\&v=2. Zugegriffen: Febr. 2020.

Donzelot J. (1980). Die Ordnung der Familie. Frankfurt a. M.: Suhrkamp.

Durham, D. (2007). Empowering youth: Making youth citizens in Botswana. In J. Cole \& D. Durham (Hrsg.), Generations and globalization (S. 102-131). Bloomington: Indiana University Press.

Durkheim, É. (1972). Erziehung und Soziologie. Düsseldorf: Schwann.

Eisenstadt, S. N. (1956). From generation to generation. Glencoe, IL: Free Press.

Entrich, S. R. (2015). The decision for shadow education in Japan: Students' choice or parents' pressure? Social Science Japan Journal, 18, 193-216.

Esengul, C. (2012). Youth and public policy in Kyrgyzstan. New York: International Debate Education Association.

Eurostat (2018). Gender pay gap statistics. http://ec.europa.eu/eurostat/statistics-explained/index.php/ Gender_pay_gap_statistics. Zugegriffen: Febr. 2020.

Gerlach, I. (1996). Familie und staatliches Handeln. Ideologie und politische Praxis in Deutschland. Opladen: Leske + Budrich.

Goody, J. (1989). Die Entwicklung von Ehe und Familie in Europa. Frankfurt a. M.: Suhrkamp.

Gruijters, R. J. (2016). Beyond filial piety. Intergenerational relations and old age security in contemporary China. Dissertation. Berlin: Humboldt-Universität zu Berlin.

Gulbrandsen, L., \& Langsether, A. (2000). Wealth distribution between generations: A source of conflict or cohesion. In S. Arber \& C. Attias-Donfut (Hrsg.), The myth of generational conflict: The family and state in ageing societies (S. 69-87). London: Routledge.

Hajnal, W. J. (1965). European marriage patterns in perspective. In D. V. Glass \& D. E. C. Everseley (Hrsg.), Population in history. Essays in historical demography, Vol. I: General and Great Britain (S. 101-146). London: Arnold.

Handrahan, L. M. (2001) Gender and ethnicity in the „Transitional Democracy“ of Kyrgyzstan. Central Asian Survey, 20, 467-496.

Hendrick, H. (1997). Children, childhood and English society, 1880-1990. Cambridge: Cambridge University Press.

Holzmann, R., \& Hinz, R. (2005). Old-age income support in the 21st century: An international perspective on pension systems and reform. Washington: The World Bank.

Hu, Y. (2018). Patriarchal hierarchy? Gender, children's housework time and family structure in postreform China. Chinese Sociological Review, 50, 310-338.

Hunner-Kreisel, C., \& Bohne, S. (Hrsg.). (2016). Childhood, youth and migration. Dodrecht: Springer.

Jacquemin, M. (2004). Children's domestic work in Abidjan, Côte d'Ivoire. The ,petites bonnes“ have the floor. Childhood, 11, 383-397.

Kaplan, M. S., Henkin, N., \& Kusano, A. T. (2002). Linking lifetimes: A global view of intergenerational exchange. Lanham: UPA.

Kağıtçıbaşı, C. (2007). Family, self, and human development across cultures. London: Routledge.

Kertzer, D. I. (1983). Generation as a sociological problem. Annual Review of Sociology, 9, 125-149.

Kim, E. (2020). Child marriage in Kygyzstan: Exploring institutional ambivalences in constructing the „,victim“. In D. Bühler-Niederberger \& L. Alberth (Hrsg.), Victim, perpetrator, or what else? Generational and gender perspectives on children, youth, and violence (S. 171-190). Bingley: Emerald.

Kim, W. S. (2008) Die Entstehung ,neuer Wohlfahrtsstaaten“ und globale Policy-Diffusion - das Beispiel Südkorea. Zeitschrift für Soziologie, 37, 186-205.

Klünder, N., \& Meier-Gräwe, U. (2017). Gleichstellung und innerfamiliale Arbeitsteilung. In Statistisches Bundesamt (Hrsg.), Wie die Zeit vergeht. Analysen zur Zeitverwendung in Deutschland (S. 65-90). Wiesbaden: Statistisches Bundesamt.

Ko, K., \& Chapman, A. (2017). Providing care to parents in Korea. Filial piety, reciprocity, and parent-child relationship quality. Innovation in Aging, 1(S1), 603. https://doi.org/10.1093/geroni/igx004.2112. Zugegriffen: Febr. 2020.

Kohli, M., Kühnemund, H., Model, A., \& Szydlik, M. (2000). Families apart? Intergenerational transfers in East and West Germany. In S. Arber \& C. Attias-Donfut (Hrsg.), The myth of generational conflict: The family and state in ageing societies (S. 88-99). London: Routledge.

Kolland, F. (2005). Globalisierung des Alterns. In A. Amann \& G. Majce (Hrsg.), Soziologie in interdisziplinären Netzwerken (S. 153-164). Köln: Böhlau.

Koyano, W. (1996). Filial piety and intergenerational solidarity in Japan. Australian Journal on Ageing, 15, 51-56. 
Lamb, S. (2009). Ageing and the Indian diaspora. Cosmopolitan families in India and abroad. Bloomington: Indiana University Press.

Lang, S. (1985). Lebensbedingungen und Lebensqualität von Kindern. Frankfurt a. M.: Mayer.

Langer-El Sayed, I. (1980). Familienpolitik: Tendenzen, Chancen, Notwendigkeiten. Frankfurt a. M.: Campus.

Lareau, A. (2003). Unequal childhoods. Berkeley: University of California Press.

LBS (Hrsg.). (2009). Wir sagen Euch mal was. LBS-Kinderbarometer, Deutschland 2009. Stimmungen, Trends und Meinungen von Kindern in Deutschland. https://www.lbs.de/media/unternehmen/west_6/ kibaro/Kibaro_2009.pdf. Zugegriffen: Febr. 2020.

Lee, W. K., \& Kwok, H. (2005). Differences in expectations and patterns of informal support for older persons in Hong Kong: Modification to filial piety. Ageing International, 30, 188-206.

Leisering, L. (Hrsg.). (2011). Die Alten der Welt. Neue Wege der Alterssicherung im globalen Norden und Süden. Frankfurt a. M.: Campus.

Leopold, T. (2018). Gender differences in the consequences of divorce. A study of multiple outcomes. Demography, 56, 769-797.

Lloyd-Sherlock, P. (2011). Ageing and international development - A critical view. In L. Leisering (Hrsg.), Die Alten der Welt. Neue Wege der Alterssicherung im globalen Norden und Süden. Frankfurt a. M.: Campus.

Lorenz-Meyer, D., \& Grotheer, A. (2000). Reinventing the generational contract. Anticipated care-giving responsibilities of younger German and Turkish migrants. In S. Arber \& C. Attias-Donfut (Hrsg.), The myth of generational conflict: The family and state in ageing societies (S. 190-208). London: Routledge.

Lu, Y. (2012). Education of children left behind in rural China. Journal of Marriage and Family, 74, 328-341.

Magazine, R., \& Ramirez Sanchez, M. A. (2007). Continuity and change in San Pedro Tlalcuapan, Mexico. In J. Cole \& D. Durham (Hrsg.), Generations and globalization (S. 52-73). Bloomington: Indiana University Press.

Mahood, L. (1995). Policing, gender, class and familiy. Britain, 1850-1940. London: UCL Press.

Mannheim, K. (1964). Das Problem der Generationen. In K. Mannheim, Wissenssoziologie (S. 509-565). Neuwied: Luchterhand.

Manuel, C. (2015). Levelling the legal play field: What the law can and can't do to improve women's access to secured finance. In F. Dahan (Hrsg.), Research handbook on secured financing in commercial transactions (S. 132-166). Cheltenham: Edward Elgar.

Markus, H. R., \& Kitayama, S. (1991). Culture and the self: Implications for cognition, emotion and motivation. Psychological Review, 98, 224-253.

Mayall, B. (2000). The sociology of childhood in relation to children's rights. The International Journal of Children's Rights, 8, 243-259.

Molchanova, E., \& Galako, T. (2017). Mental health consequences of bride kidnapping in the Kyrgyz Republic. European Psychiatry, 41, S515.

Muldoon, J. C., Williams, J. C., \& Lawrence, A. (2014). „Mum cleaned it and I just played with it.“ Children's perceptions and responsibilities in the care of family pets. Childhood, 22, 201-216.

Nauck, B. (2007). Value of children and the framing of fertility. Results from a cross-cultural survey in 10 societies. European Sociological Review, 23, 615-629.

Nave-Herz, R. (2015). Familie heute. Darmstadt: Wissenschaftliche Buchgesellschaft.

Ostner, I., Betz, T., \& Honig, M.-S. (2017). Introduction: Parenting practices and parenting support in recent debates and policies. In T. Betz, M.-S. Honig \& I. Ostner (Hrsg.), Parents in the spotlight: Parenting practices and support from a comparative perspective (S. 5-22). Journal of Family Research, Sonderheft 11. Opladen: Verlag Barbara Budrich.

Parsons, T., \& Bales, R. F. (1955). Familiy, socialization and interaction process. New York: Free Press.

Pascall, G., \& Manning, N. (2000). Gender and social policy: Comparing welfare states in Central and Eastern Europe and the former Soviet Union. Journal of European Social Policy, 10, 240-266.

Punch, S. (2001). Household division of labour: Generation, gender, age, birth order and sibling composition. Work, Employment and Society, 15, 803-823.

Punch, S., \& Vanderbeck, R.M. (Hrsg.). (2018). Families, intergenerationality, and peer group relations. Dordrecht: Springer.

Qi, X. (2015). Filial obligation in contemporary China: Evolution of the culture-system. Journal for the Theory of Social Behaviour, 45, 141-161.

Roussel, L. (1976). La famille après le mariage des enfants. Les relations entre générations. Population, 31, 1195-1206. 
Roussel, L., \& Bourguignon, O. (1975). La famille après le mariage des enfants. Paris: Presses Universitaires de France.

Schaub, M., Henck, A., \& Baker, D. P. (2017). The globalized ,whole child“: Cultural understandings of children and childhood in multilateral aid development policy, 1946-2010. Comparative Education Review, 16, 298-326.

Schlumbohm, J. (1983). Kinderstuben. Wie Kinder zu Bauern, Bürgern, Aristokraten wurden. München: dtv.

Schneider, N. F., Diabaté, S., \& Ruckdeschel, K. (2015). Familienleitbilder in Deutschland. Opladen: Verlag Barbara Budrich.

Schwittek, J. (2017). Double Facework. Aushandlungsprozesse junger Erwachsener in Kirgistan zwischen Selbst und Kollektiv. Opladen: Verlag Barbara Budrich.

Segalen, M. (1990). Die Familie. Geschichte, Soziologie, Anthropologie. Frankfurt a. M.: Campus.

Sharma, K., \& Kemp, C. L. (2012). „One should follow the wind“: Individualized filial piety and support exchanges in Indian immigrant families in the United States. Journal of Aging Studies, 26, 129-139.

Shin, C. (2003). Social policy in South Korea: Cultural and structural factors in the emergence of welfare. Social Policy Administration, 37, 328-341.

De Singly, F. (2005). Le soi, le couple et la famille. Paris: Nathan.

Skevik, A. (2003). Children of the welfare state. Individuals with entitlements, or hidden in the family. Journal of Social Policy, 32, 423-440.

Sommerkorn, I. (1988). Die erwerbstätige Mutter in der Bundesrepublik. Einstellungs- und Problemveränderungen. In R. Nave-Herz (Hrsg.), Wandel und Kontinuität der Familie in der Bundesrepublik Deutschland (S. 115-144). Stuttgart: Enke.

Sung, S. (2003). Women reconciling paid and unpaid work in a Confucian welfare state: The case of South Korea. Social Policy Administration, 37, 342-360.

Szydlik, M. (2016). Sharing lives: Adult children and parents. London: Routledge.

Triandis, H. C. (2001). Individualism-collectivism and personality. Journal of Personality, 69, 907-924.

UNDP (2010). Kyrgyzstan: Successful youth - successful country. NHDR Report. Bishkek: UNDP.

Vincent, C., \& Ball, S. J. (2007). „Making up“ the middle class child: Families, activities and class dispositions. Sociology, 41, 1061-1077.

Walper, S., Fichtner, J., \& Normann, K. (2013) Hochkonflikthafte Trennungsfamilien. Weinheim: Juventa.

Williamson, J. B., Price, M., \& Shen, C. (2011). Pension policy in China, Singapore and South Korea: An assessment of the potential of the notional defined contribution model. Journal of Aging Studies, 26, 79-89.

Woldehanna, T., Jones, N., \& Tefera, B. (2008). The invisibility of children's paid and unpaid work. Implications for Ethiopia's national poverty reduction policy. Childhood, 15, 177-202.

World Bank (1994). Averting the old age crisis. http://documents.worldbank.org/curated/en/ 973571468174557899/pdf/multi-page.pdf. Zugegriffen: Febr. 2020.

World Bank (2011). Learning for all - Education strategy 2020. Washington: The International Bank for Reconstruction and Development. http://pubdocs.worldbank.org/en/418511491235420712/ Education-Strategy-4-12-2011.pdf. Zugegriffen: Febr. 2020.

World Bank (o.J.). Migration and remittances data. http://www.worldbank.org/en/topic/ migrationremittancesdiasporaissues/brief/migration-remittances-data. Zugegriffen: Febr. 2020.

World Vision Deutschland (Hrsg.). (2018). Kinder in Deutschland. 4. World Vision Kinderstudie. Weinheim: Beltz.

Woronow, T. E. (2007). Chinese children, American education. In J. Cole \& D. Durham (Hrsg.), Generations and globalization (S. 29-51). Bloomington: Indiana University Press.

Yeh, K.-H., Yi, C.-C., Tsao, W.-C., \& Wan, P. S. (2013). Filial piety in contemporary Chinese societies. A comparative study of Taiwan, Hong Kong and China. International Sociology, 28, 277-296.

Zelizer, V., (1985). Pricing the priceless child. The changing social value of children. Princeton: Princeton University Press.

Zinnecker, J., \& Silbereisen, R. (1996). Kindheit in Deutschland. München: Juventa.

Zinnecker, J., Behnken, I., Stecher, L., \& Maschke, S. (2003). Null zoff \& voll busy. Die erste Jugendgeneration des neuen Jahrhunderts. Opladen: Leske + Budrich. 
Doris Bühler-Niederberger geb. 1950. Dr. Phil., Professorin für Soziologie an der Bergischen Universität Wuppertal. Forschungsschwerpunkte: Soziologische Analyse des Aufwachsens und der Kindheit, insbesondere Fragen nach sozialer Ungleichheit und nach Institutionen und Mustern der Kindheit zwischen lokalen Bedingungen und globalen Entwicklungen. Wichtige Veröffentlichungen: Lebensphase Kindheit, 2020; Marginality and voice - Childhood in sociology and society, in: Current Sociology, 2010; Success - a „leitmotiv“ of Kyrgyz childhood, in: C. Baraldi \& L. Rabello de Castro (Hrsg.), Global childhoods in international perspective, 2020; (Hrsg., mit L. Alberth) Victim, perpetrator, or what else? Generational and gender perspectives on children, youth, and violence, 2020. 